\title{
Convergence in the $p$-Contest
}

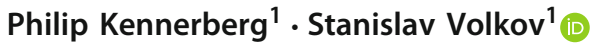

Received: 26 July 2019 / Accepted: 14 January 2020 / Published online: 24 January 2020

(c) The Author(s) 2020

\begin{abstract}
We study asymptotic properties of the following Markov system of $N \geq 3$ points in $[0,1]$. At each time step, the point farthest from the current centre of mass, multiplied by a constant $p>$ 0 , is removed and replaced by an independent $\zeta$-distributed point; the problem, inspired by variants of the Bak-Sneppen model of evolution and called a p-contest, was posed in Grinfeld et al. (J Stat Phys 146, 378-407, 2012). We obtain various criteria for the convergences of the system, both for $p<1$ and $p>1$. In particular, when $p<1$ and $\zeta \sim U[0,1]$, we show that the limiting configuration converges to zero. When $p>1$, we show that the configuration must converge to either zero or one, and we present an example where both outcomes are possible. Finally, when $p>1, N=3$ and $\zeta$ satisfies certain mild conditions (e.g. $\zeta \sim U[0,1]$ ), we prove that the configuration converges to one a.s. Our paper substantially extends the results of Grinfeld et al. (Adv Appl Probab 47:57-82, 2015) and Kennerberg and Volkov (Adv Appl Probab 50:414-439, 2018) where it was assumed that $p=1$. Unlike the previous models, one can no longer use the Lyapunov function based just on the radius of gyration; when $0<p<1$ one has to find a more finely tuned function which turns out to be a supermartingale; the proof of this fact constitutes an unwieldy, albeit necessary, part of the paper.
\end{abstract}

Keywords Keynesian beauty contest · Jante's law $\cdot$ Rank-driven process

Mathematics Subject Classification Primary 60J05; Secondary 60D05 · 60F15 · 60K35 . $82 \mathrm{C} 22 \cdot 91 \mathrm{~A} 15$

\section{Introduction}

This paper extends the results of [3,4] on the so-called Keynesian beauty contest, or, as it was called in [4], Jante's law process. Following [3], we recall that in the Keynesian beauty

Communicated by Eric A. Carlen.

The research is partially supported by the Swedish Research Council Grant VR 2014-5157 and by Crafoord foundation Grant 20190667.

$凶 \quad$ Stanislav Volkov

S.Volkov@maths.lth.se

1 Centre for Mathematical Sciences, Lund University, Box 118, 22100 Lund, Sweden 
contest, we have $N$ players guessing a number, and the person who guesses closest to the mean of all the $N$ guesses wins; see [5, Ch. 12, Sect. V]. The formal version, suggested by Moulin [6, p. 72], assumes that this game is played by choosing numbers on the interval $[0,1]$, the " $p$-beauty contest", in which the target is the mean value, multiplied by a constant $p>0$. For the applications of the $p$-contest in the game theory, we refer the reader to e.g. [7]; see also [1,3] and references therein for further applications and other relevant papers.

The version of the $p$-contest with $p \equiv 1$ was studied in [3,4]. In [3] it was shown that in the model where at each unit of time the point farthest from the center of mass is replaced by a point chosen uniformly on $[0,1]$, then eventually all (but one) points converge almost surely to some random limit the support of which is the whole interval $[0,1]$; many of the results were extended for the version of the model on $\mathbb{R}^{d}, d \geq 2$. The results of [3] were further generalized in [4], by removing the assumption that a new point is chosen uniformly on $[0,1]$, as well as by removing more than one point at once, these points being chosen in such a way that the moment of inertia of the resulting configuration is minimized. However, the case $p \neq 1$ was not addressed in either of these two papers.

Let us now formally define the model; the notation will be similar to those in [3,4]. Let $\mathcal{X}=$ $\left\{x_{1}, x_{2}, \ldots, x_{N}\right\} \in \mathbb{R}^{N}$ be an unordered $N$-tuple of points in $\mathbb{R}$, and $\left(x_{(1)}, x_{(2)}, \ldots, x_{(N)}\right)$ be these points put in non-decreasing order, that is, $x_{(1)} \leq x_{(2)} \leq \cdots \leq x_{(N)}$. As in [3,4] let us define the barycentre of the configuration as

$$
\mu_{N}\left(x_{1}, \ldots, x_{N}\right):=N^{-1} \sum_{i=1}^{N} x_{i} .
$$

Fix some $p>0$ and also define the $p$-centre of mass as $p \mu_{N}\left(x_{1}, \ldots, x_{N}\right)$.

The point, farthest from the $p$-centre of mass, is called the extreme point of $\mathcal{X}$, and it can be either $x_{(1)}$ or $x_{(N)}$ (with possibility of a tie), and the core of $\mathcal{X}$, denoted by $\mathcal{X}^{\prime}$, is constructed from $\mathcal{X}$ by removing the extreme point; in case of a tie between the left-most and the right-most point, we choose either of them with equal probability (same as in $[3,4]$ ). Throughout the rest of the paper, $x_{(1)}(t), \ldots, x_{(N-1)}(t)$ shall denote the points of the core ${ }^{1}$ $\mathcal{X}^{\prime}(t)$ put into non-decreasing order.

Our process runs as follows. Let $\mathcal{X}(t)=\left\{X_{1}(t), \ldots, X_{N}(t)\right\}$ be an unordered $N$-tuple of points in $\mathbb{R}$ at time $t=0,1,2, \ldots$ Given $\mathcal{X}(t)$, let $\mathcal{X}^{\prime}(t)$ be the core of $\mathcal{X}(t)$ and replace $\mathcal{X}(t) \backslash \mathcal{X}^{\prime}(t)$ by a $\zeta$-distributed random variable so that

$$
\mathcal{X}(t+1)=\mathcal{X}^{\prime}(t) \cup\left\{\zeta_{t+1}\right\},
$$

where $\zeta_{t}, t=1,2, \ldots$, are i.i.d. random variables with a common distribution $\zeta$.

Finally, to finish the specification of our process, we allow the initial configuration $\mathcal{X}(0)$ to be arbitrary or random, with the only requirement being that all the points of $\mathcal{X}(0)$ must lie in the support of $\zeta$.

Throughout the paper we will use the notation $A \underset{\text { a.s. }}{\Longrightarrow} B$ for two events $A$ and $B$, whenever $\mathbb{P}\left(A \cap B^{c}\right)=0$, that is, when $A \subseteq B$ up to a set of measure 0. We will also write, with some abuse of notations, that $\lim _{t \rightarrow \infty} \mathcal{X}^{\prime}(t)=a \in \mathbb{R}$ or equivalently $\mathcal{X}^{\prime}(t) \rightarrow a$ as $t \rightarrow \infty$ if $\mathcal{X}^{\prime}(t) \rightarrow(a, a, \ldots, a) \in \mathbb{R}^{N-1}$, i.e. $\lim _{t \rightarrow \infty} x_{(i)}(t)=a$ for all $i=1,2, \ldots, N-1$. Similarly, for an interval $(a, b)$ we will write $\mathcal{X}^{\prime}(t) \in(a, b)$ whenever all $x_{(1)}(t), \ldots, x_{(N-1)}(t) \in(a, b)$. Finally, we will assume that inf $\emptyset=+\infty$, and use the notation $y^{+}=\max (y, 0)$ for $y \in \mathbb{R}$.

1 Rather than of $\mathcal{X}(t)$ 
Also we require that $\zeta$ has a full support on $[0,1]$, that is, $\mathbb{P}(\zeta \in(a, b))>0$ for all $a, b$ such that $0 \leq a<b \leq 1$.

\section{The Case $p<1$}

Throughout this Section we assume that $0<p<1$ and that $\operatorname{supp} \zeta=[0,1]$. Because of the scaling invariance, our results may be trivially extended to the case when $\operatorname{supp} \zeta=[0, A]$, $A \in(0, \infty)$; some of them are even true when $A=\infty$; however, to simplify the presentation from now on we will deal only with the case $A=1$.

First, we present some general statements; more precise results will follow in case where $\zeta \sim U[0,1]$.

\section{Proposition 1 We have}

(a) $\liminf \inf _{t \rightarrow \infty} x_{(N-1)}(t)=0$;

(b) $\mathbb{P}\left(\exists \lim _{t \rightarrow \infty} \mathcal{X}^{\prime}(t) \in(0,1]\right)=0$;

(c) if $p<\frac{1}{2}+\frac{1}{2(N-1)}$ then $\mathbb{P}\left(\lim _{t \rightarrow \infty} \mathcal{X}^{\prime}(t)=0\right)=1$;

(d) if $p<\frac{1}{2}+\frac{1}{N-2}$ then $\left\{x_{(1)}(t) \rightarrow 0\right\} \underset{\text { a.s. }}{\Longrightarrow}\left\{\lim _{t \rightarrow \infty} \mathcal{X}^{\prime}(t)=0\right\}$.

Proof (a) Since $\zeta$ has full support on $[0,1]$ it follows that (see [4, Proposition 1]) there exists a function $f: \mathbb{R}^{+} \rightarrow \mathbb{R}^{+}$such that

$$
\mathbb{P}(\zeta \in(a, b)) \geq f(b-a)>0 \text { for all } 0 \leq a<b \leq 1 .
$$

Also, to simplify notations, we write $\mu=\mu_{N}(\mathcal{X}(t))$ throughout the proof.

Fix a small positive $\varepsilon$ such that $p+2 \varepsilon<1$. Suppose that for some $t$ we have $x_{(N-1)}(t) \leq$ $b \leq 1$. We will show that $x_{(N-1)}(t+N) \leq b(1-\varepsilon)$ with a strictly positive probability which only depends on $p, b, \epsilon$ and $N$. Assume that we have $\zeta_{t+1}, \ldots, \zeta_{t+N-1} \in(p b,(p+\varepsilon) b) \subset$ $(p b, b)$; this happens with probability no less than $[f(p \varepsilon b)]^{N-1}$. We claim that by the time $t+N$ we have $x_{(N-1)}(t+N-1)<(p+\varepsilon) b$. Indeed, $p \mu \leq p b$ always lies to the left of the newly sampled points, therefore either there are no more points to the right of $(p+\varepsilon) b$ at some time $s \in[t, t+N-1]$ (which implies that there will be no points there at time $t+N$ due to the sampling range of the new points), or one of the older points, i.e. present at time $t$, gets removed (it can be the one to the left of $p b$ ). Since we eventually have to replace all the $N-1$ old points, then $x_{(N-1)}(t+N) \leq b(1-\varepsilon)$.

Fix a $\delta>0$ and find $M$ so large that $(1-\varepsilon)^{M}<\delta$. Let the event $C(s)=$ $\left\{x_{(N-1)}(s)<\delta\right\}$. By iterating the above argument, we get that $\mathbb{P}\left(C(t+N M) \mid \mathcal{F}_{t}\right) \geq$ $\prod_{i=1}^{M}\left[f\left(p \varepsilon(1-\varepsilon)^{i-1}\right)\right]^{N-1}$, since at time $t$ we can set $b=1$. Therefore, $\sum_{m} \mathbb{P}(C(N M(m+$ 1)) $\left.\mid \mathcal{F}_{N M m}\right)=\infty$ and by Lévy's extension of the Borel-Cantelli lemma (see e.g. [8]) infinitely many $C(s)$ occur. Since $\delta>0$ is arbitrary, we get $\liminf _{t \rightarrow \infty} x_{(N-1)}(t)=0$.

(b) Let $r=\frac{1+p^{-1}}{2}>1$. Suppose that the core converges to some point $x \in(0,1]$; then there exist a rational $q \in(0,1]$ and a $T>0$ such that $\mathcal{X}^{\prime}(t) \in(q, r q)$ for all $t \geq T$, formally

$$
\left\{\exists \lim \mathcal{X}^{\prime}(t) \in(0,1]\right\} \subseteq \bigcup_{q \in Q \cap(0,1]} \bigcup_{T>0} \bigcap_{t \geq T} A_{q, t}
$$

where $A_{q, t}=\left\{\mathcal{X}^{\prime}(t) \in(q, r q)\right\}$. We will show that

$$
\mathbb{P}\left(A_{q, t+1} \mid \mathcal{F}_{t}, A_{q, t}\right)<1-v_{q} \text { for all } t
$$


for some $v_{q}>0$. This will imply, in turn, that

$$
\mathbb{P}\left(\bigcap_{t \geq T} A_{q, t}\right)=0
$$

and hence the RHS (and thus the LHS as well) of (2.2) has the probability 0 .

Suppose $A_{q, t}$ has occurred and the newly sampled point $\zeta \in(p q, q)$. Then

$$
p \mu_{N}\left(\mathcal{X}^{\prime}\left(\tau_{k}\right) \cup\{\zeta\}\right)<\operatorname{prq}=\frac{p q+q}{2}<\frac{\zeta+x_{(N-1)}}{2}
$$

Consequently, $x_{(N-1)}$ lies further from the $p$-center of mass, and hence it should be removed. The new configuration will, however, contain the point $\zeta \notin(q, r q)$ and hence $A_{q, t+1}$ does not occur. Thus

$$
\mathbb{P}\left(A_{q, t+1} \mid \mathcal{F}_{t}, A_{q, t}\right) \leq 1-\mathbb{P}(\zeta \in(q, r q)) \leq 1-f(p q-q)=: 1-v_{q}
$$

as required.

(c) First, we will show that it is the right-most point of the configuration which should be always removed; note that it suffices to check this only when $x_{(N)}>0$. Indeed, by the assumption on $p$ we have

$$
\mu \leq \frac{(N-1) x_{(1)}+(N-1) x_{(N)}}{N}=\frac{2 p(N-1)}{N} \cdot \frac{x_{(1)}+x_{(N)}}{2 p}<\frac{x_{(1)}+x_{(N)}}{2 p}
$$

implying that

$$
x_{(N)}-p \mu>p \mu-x_{(1)} \Longleftrightarrow x_{(N)}-p \mu>\left|p \mu-x_{(1)}\right|
$$

Therefore, $x_{(N)}$ is the farthest point from the $p$-centre of mass. This implies that $x_{(N-1)}(t)$ is non-increasing and therefore result now easily follows from part (a) since $x_{(N-1)}(t)$ is an upper bound for all the core points.

(d) Apply Corollary 3 with $k=1$; it is possible because of Remark 3 .

We are ready to present the main result of this Section.

Theorem 1 Suppose that $\zeta \sim U[0,1]$. Then $\mathcal{X}^{\prime}(t) \rightarrow 0$ a.s.

Proof Proposition 1(c) implies that we now only need to consider the case $p \geq \frac{N}{2(N-1)}$, which we will assume from now on.

Let us introduce a modification of this process on $[0,+\infty)$ which we will call the borderless p-contest; it is essentially the same process as the one in [3, Sect. 3.4]. In order to do this, we need the following statement.

Lemma 1 Suppose that $x_{1}, \ldots, x_{N-1}>0$. Then there exists an $R=R\left(x_{(N-1)}\right) \geq 0$ such that $x$ is the farthest point from $p \mu=\frac{p}{N}\left(x_{1}+\cdots+x_{N-1}+x\right)$ whenever $x>R$.

Proof of Lemma 1 Set $R=6 x_{(N-1)}$. Then $x>x_{(1)}$ is farther from the centre of mass than $x_{(1)}$ if and only if

$$
\begin{gathered}
x-p \mu>\left|p \mu-x_{(1)}\right| \Longleftrightarrow x-p \mu>p \mu-x_{(1)} \\
\Longleftrightarrow x\left(1-\frac{2 p}{N}\right)>2 p \frac{x_{1}+\cdots+x_{N-1}}{N}-x_{(1)}
\end{gathered}
$$


This is true, due to the fact that $x>R$ and

$$
x\left(1-\frac{2 p}{N}\right)>\frac{x}{3}>2 x_{(N-1)}>2 p x_{(N-1)}>2 p \frac{x_{1}+\cdots+x_{N-1}}{N}
$$

since $p<1$ and $N \geq 3$.

The borderless process is constructed as follows. Our core configuration starts as before in $[0,1]$, and we use the same rejection/acceptance criteria for new points. However, we will now allow points to be generated to the right of 1 as well. Let $R_{t}=R\left(x_{(N-1)}(t)\right)$ where $R$ is taken from Lemma 1 . Then a new point is sampled uniformly and independently of the past on the interval $\left[0, R_{t}\right]$; formally, it is given by $R_{t} U_{t}$ where $U_{t}$ are i.i.d. uniform $[0,1]$ random variables independent of everything. Observe that if we consider the embedded process only at the times when the core configuration changes, then the exact form of the function $R(\cdot)$ is irrelevant, due to the fact that the uniform distribution conditioned on a subinterval is also uniform on that subinterval.

Next, for $y=\left\{y_{1}, \ldots, y_{N-1}\right\}$ define the function

$$
h(y)=F(y)+k \mu(y)^{2},
$$

where

$$
F(y)=\sum_{i=1}^{N-1}\left(y_{i}-\mu(y)\right)^{2}, \quad \mu(y)=\frac{1}{N-1} \sum_{i=1}^{N-1} y_{i}, \quad k=\frac{(N-1)^{2}(1-p)}{N-2} .
$$

We continue with the following

Lemma 2 For the borderless $p$-contest the sequence of random variables $h\left(\mathcal{X}^{\prime}(t)\right) \geq 0$, $t=1,2, \ldots$, is a supermartingale.

Remark 1 Note that the function $F(\cdot)$ defined above is a Lyapunov function for the process in [3]; this is no longer the case as long as $p \neq 1$; that is why we have to use a carefully chosen "correction" factor which involves the barycentre of the configuration.

Proof of Lemma 2 Assume that $x_{(N-1)}(t)>0$ (otherwise the process has already stopped, and the result is trivial). The inequality, which we want to obtain is

$$
\left.\mathbb{E}\left[h\left(\mathcal{X}^{\prime}(t+1)\right)-h\left(\mathcal{X}^{\prime}(t)\right) \mid \mathcal{F}_{t}\right]\right|_{x(t)=y} \leq 0
$$

for all $y=\left(y_{1}, \ldots, y_{N-1}\right)$ with $y_{i} \in[0,1]$. Note that the function $h(y)$ is homogeneous of degree 2 in $y$, therefore w.l.o.g. we can assume that $\max y \equiv 1$.

For simplicity let $M=N-1 \geq 2$, and let

$$
z=6 U_{t} \text { (the newly sampled point), } \quad a=\min y<1 \text { (the leftmost point) }
$$

Note also that

$$
p \geq \frac{N}{2(N-1)}=\frac{M+1}{2 M}=\frac{1}{2}+\frac{1}{2 M} .
$$

Define

$$
\begin{aligned}
F_{\text {old }} & =F(y), & F_{\text {new }} & =F\left((y \cup\{z\})^{\prime}\right) \\
\mu_{\text {old }}^{\prime} & =\mu(y), & \mu_{\text {new }}^{\prime} & =\mu\left((y \cup\{z\})^{\prime}\right), \\
h_{\text {old }} & =F_{\text {old }}+k\left(\mu_{\text {old }}^{\prime}\right)^{2}, & h_{\text {new }} & =F_{\text {new }}+k\left(\mu_{\text {new }}^{\prime}\right)^{2}
\end{aligned}
$$


Thus we need to establish

$$
\mathbb{E}\left[h_{\text {new }}-h_{\text {old }} \mid \mathcal{F}_{t}\right] \leq 0 .
$$

First of all, observe that if $\tilde{y}=\left(y \backslash\left\{y_{i}\right\}\right) \cup\{z\}$, that is, $\tilde{y}$ is obtained from $y$ by replacing $y_{i}$ with $y_{0}$, then

$$
\begin{aligned}
F(\tilde{y})-F(y) & =\frac{z-y_{i}}{M}\left[(M-1) z+(M+1) y_{i}-2 M \mu(y)\right] \\
\mu(\tilde{y})^{2}-\mu(y)^{2} & =\frac{z-y_{i}}{M^{2}}\left[z-y_{i}+2 M \mu(y)\right]
\end{aligned}
$$

In particular, if we replace point $a$ by the new point $z$, then

$$
\begin{aligned}
\Delta_{a}(z) & :=h_{\text {new }}-h_{\text {old }} \\
& =\frac{z-a}{M}\left[(M-1) z+(M+1) a-2 M \mu(y)+\frac{k}{M}(z-a+2 M \mu(y))\right]
\end{aligned}
$$

and if we replace point 1 , then

$$
\begin{aligned}
\Delta_{1}(z) & :=h_{\text {new }}-h_{\text {old }} \\
& =\frac{z-1}{M}\left[(M-1) z+(M+1)-2 M \mu(y)+\frac{k}{M}(z-1+2 M \mu(y))\right]
\end{aligned}
$$

Note that both $\Delta_{a}$ and $\Delta_{1}$ depend only on four variables $(a, z, \mu, M)$ but not the whole configuration. Let us also define

$$
m(z)=p \cdot \frac{y_{1}+\cdots+y_{M}+z}{M+1}=p \cdot \frac{M \mu+z}{M+1},
$$

the $p$-centre of mass of the old core and the newly sampled point.

There are three different cases that can occur: either (a) the point $a$ is removed, (b) 1 , the rightmost point of the previous core, is removed, or (c) the newly sampled point $z$ is removed. In the third case the core remains unchanged, and the change in the value of the function $h$ is trivially zero. The point $a$ can only be removed if $z>a$; the point 1 can only be removed if $z<1$; the point $z$ can be possibly removed only if $z \in(0, a)$ or $z \in(1, \infty)$. Let us compute the critical values for $z$, for which there is a tie between the farthest points.

\section{Which Point to Remove?}

(i) Suppose $z<a$. Then there is a tie between $z$ and 1 if and only if $m(z)=\frac{z+1}{2}$, that is if

$$
z=t_{z 1}:=\frac{M(2 p \mu-1)-1}{M+1-2 p} \in \begin{cases}(-\infty, 0) & \text { if } p<p_{1}:=\frac{M+1}{2 M \mu} \\ (0, a) & \text { if } p_{1}<p<p_{2}:=\frac{(M+1)(a+1)}{2 M \mu+2 a} \\ (a,+\infty) & \text { if } p>p_{2} .\end{cases}
$$

Thus, we have:

- when $p<p_{1}$, point 1 is removed;

- when $p_{1}<p<p_{2}$, if $z<t_{z 1}$ then $z$ is removed; if $z>t_{z 1}$ point 1 is removed;

- when $p>p_{2}$, point $z$ is removed. 
(ii) Suppose $a<z<1$. There is a tie between $a$ and 1 if and only if $m(z)=\frac{a+1}{2}$, that is if

$$
z=t_{a 1}:=\frac{(M+1)(a+1)-2 M \mu p}{2 p} \in \begin{cases}(1,+\infty) & \text { if } p<p_{3}:=\frac{(M+1)(a+1)}{2 M \mu+2}, \\ (a, 1) & \text { if } p_{3}<p<p_{2}, \\ (-\infty, a) & \text { if } p>p_{2} .\end{cases}
$$

Thus, we have:

- when $p<p_{3}$, point 1 is removed;

- when $p_{3}<p<p_{2}$, if $z<t_{a 1}$ then 1 is removed; if $z>t_{a 1}$ then point $a$ is removed;

- when $p>p_{2}$, point $a$ is removed.

(iii) Suppose $z>1$. There is a tie between $z$ and $a$ if and only if $m(z)=\frac{z+a}{2}$, that is if

$$
z=t_{z a}:=\frac{2 M \mu p-(M+1) a}{M+1-2 p} \in \begin{cases}(-\infty, 1) & \text { if } p<p_{3}, \\ (1,+\infty) & \text { if } p>p_{3} .\end{cases}
$$

Thus, we have:

- when $p<p_{3}$, point $z$ is removed;

- when $p>p_{3}$, if $z<t_{z a}$ then $a$ is removed; if $z>t_{z a}$ then point $z$ is removed.

We always have $p_{1}<p_{2}, p_{3}<p_{2}$ since

$$
\begin{aligned}
& p_{2}-p_{1}=\frac{a(M+1)(M \mu-1)}{2 M \mu(M \mu+a)}=\frac{a(M+1)(a+(M-2) f)}{2 M \mu(M \mu+a)}>0, \\
& p_{2}-p_{3}=\frac{(1-a)^{2}(M+1)}{2(M \mu+1)(M \mu+a)}>0,
\end{aligned}
$$

while

$$
p_{1}<p_{3} \Longleftrightarrow M a \mu>1 \Longleftrightarrow f>\frac{1-a-a^{2}(M-1)}{a(M-2)(1-a)}(\text { when } M>2)
$$

The final observation is that $t_{z a}<6$, so there is indeed no need to sample the new point outside of the range $(0,6)$; this holds since $M \geq 2$ and

$$
\begin{aligned}
6-t_{z a} & =\frac{-2 p(M \mu+6)+M a+6 M+a+6}{M+1-2 p}>\frac{-2 M \mu+M a+6 M+a-6}{M+1-2 p} \\
& >\frac{-2 M \mu+6 M-6}{M+1-2 p}=\frac{2 M(1-\mu)+4 M-6}{M+1-2 p}>\frac{2}{M+1-2 p}>0 .
\end{aligned}
$$

\section{The Five Cases for the Removal}

- $p<\min \left\{p_{1}, p_{3}\right\}$ :

- when $z<1$, point 1 is removed

- when $z>1$, point $z$ is removed

- $p>p_{2}$ :

- when $z<a$ or $z>t_{z a} \in(1, \infty)$ point $z$ is removed

- when $a<z<t_{z a}$, point $a$ is removed

- $\max \left\{p_{1}, p_{3}\right\}<p<p_{2}$ 
- when $z<t_{z 1} \in(0, a)$ or $t>t_{z a} \in(1,+\infty)$, point $z$ is removed

- when $t_{z 1}<z<t_{a 1} \in(a, 1)$, point 1 is removed

- when $t_{a 1}<z<t_{z a}$, point $a$ is removed

- $p_{1}<p<p_{3}\left(<p_{2}\right)$ :

- when $z<t_{z 1} \in(0, a)$ or $z>1$, point $z$ is removed

- when $t_{z 1}<z<1$, point 1 is removed

- $p_{3}<p<p_{1}\left(<p_{2}\right)$ :

- when $z<t_{a 1} \in(a, 1)$, point 1 is removed

- when $t_{a 1}<z<t_{z a} \in(1,+\infty)$, point $a$ is removed

- when $z>t_{z a}$, point $z$ is removed

Let

$$
\begin{aligned}
& X_{1}=p-p_{1}=\frac{M(2 \mu p-1)-1}{2 M \mu}, \\
& X_{2}=p-p_{2}=\frac{2 a p-a-1+(2 \mu p-a-1) M}{2(M \mu+a)}, \\
& X_{3}=p-p_{3}=\frac{2 p-a-1+(2 \mu p-a-1) M}{2(M \mu+1)} .
\end{aligned}
$$

Define

$$
\begin{aligned}
& \tilde{\mathbf{I}}_{1}=\left.\mathbb{E}\left(h\left(\mathcal{X}^{\prime}(t+1)\right)-h\left(\mathcal{X}^{\prime}(t)\right) \mid \mathcal{F}_{t}\right)\right|_{x(t)=y} \cdot 1_{X_{1}<0} \cdot 1_{X_{3}<0}, \\
& \tilde{\mathbf{I}}_{2}=\left.\mathbb{E}\left(h\left(\mathcal{X}^{\prime}(t+1)\right)-h\left(\mathcal{X}^{\prime}(t)\right) \mid \mathcal{F}_{t}\right)\right|_{x(t)=y} \cdot 1_{X_{2}>0}, \\
& \tilde{\mathbf{I}}_{3}=\left.\mathbb{E}\left(h\left(\mathcal{X}^{\prime}(t+1)\right)-h\left(\mathcal{X}^{\prime}(t)\right) \mid \mathcal{F}_{t}\right)\right|_{x(t)=y} \cdot 1_{X_{2}<0} \cdot 1_{X_{1}>0} \cdot 1_{X_{3}>0}, \\
& \tilde{\mathbf{I}}_{4}=\left.\mathbb{E}\left(h\left(\mathcal{X}^{\prime}(t+1)\right)-h\left(\mathcal{X}^{\prime}(t)\right) \mid \mathcal{F}_{t}\right)\right|_{x(t)=y} \cdot 1_{X_{1}>0} \cdot 1_{X_{3}<0}, \\
& \tilde{\mathbf{I}}_{5}=\left.\mathbb{E}\left(h\left(\mathcal{X}^{\prime}(t+1)\right)-h\left(\mathcal{X}^{\prime}(t)\right) \mid \mathcal{F}_{t}\right)\right|_{x(t)=y} \cdot 1_{X_{1}<0} \cdot 1_{X_{3}>0} .
\end{aligned}
$$

Since max $y=1$, because of the comment on the restriction of the uniform distribution on a subinterval, we have $\tilde{\mathbf{I}}_{j}=c_{j} \mathbf{I}_{j}, j=1,2,3,4,5$, where $c_{j}$ 's are some positive constants and

$$
\begin{aligned}
& \mathbf{I}_{1}=\mathbf{A}_{1} \cdot 1_{X_{1}<0} \cdot 1_{X_{3}<0}, \quad \mathbf{A}_{1}=\int_{0}^{1} \Delta_{1} d z, \\
& \mathbf{I}_{2}=\mathbf{A}_{2} \cdot 1_{X_{2}>0}, \quad \mathbf{A}_{2}=\int_{a}^{t_{z a}} \Delta_{a} d z, \\
& \mathbf{I}_{3}=\mathbf{A}_{3} \cdot 1_{X_{2}<0} \cdot 1_{X_{1}>0} \cdot 1_{X_{3}>0}, \mathbf{A}_{3}=\int_{t_{z 1}}^{t_{a 1}} \Delta_{1} d z+\int_{t_{a 1}}^{t_{z a}} \Delta_{a} d z, \\
& \mathbf{I}_{4}=\mathbf{A}_{4} \cdot 1_{X_{1}>0} \cdot 1_{X_{3}<0}, \quad \mathbf{A}_{4}=\int_{t_{z 1}}^{1} \Delta_{1} d z, \\
& \mathbf{I}_{5}=\mathbf{A}_{5} \cdot 1_{X_{1}<0} \cdot 1_{X_{3}>0}, \quad \mathbf{A}_{5}=\int_{0}^{t_{a 1}} \Delta_{1} d z+\int_{t_{a 1}}^{t_{z a}} \Delta_{a} d z .
\end{aligned}
$$

Thus to establish (2.5), it suffices to show that $\mathbf{I}_{j} \leq 0$ for each $j=1,2,3,4,5$. This is done by very extensive and tedious calculations, which can be found in the Appendix.

We now return to our original $p$-contest process $\mathcal{X}(t)$. For $L \geq 2$ define

$$
\tau_{L}=\inf \left\{t>0: x_{(N-1)}(t)<1 / L\right\}
$$




$$
\eta_{L}=\inf \left\{t>\tau_{L}: x_{(N-1)}(t) \geq 1 / 2\right\},
$$

note that $\tau_{L}$ is a.s. finite for every $L$ by Proposition 1 . Let $W(s)=\left\{w_{1}(s), \ldots, w_{N}(s)\right\}$ be a borderless $p$-contest with $W(0)=\mathcal{X}\left(\tau_{L}\right)$; let $W^{\prime}(s)$ be its core. By Lemma 2 the quantity $\xi_{t}=h\left(W^{\prime}\left(t \wedge \eta_{L}\right)\right)$ is a supermartingale, that converges to some $\xi_{\infty}$. Since $\xi_{t}$ is bounded,

$$
\begin{aligned}
\mathbb{E} \xi_{0} & \geq \mathbb{E} \xi_{\infty}=\mathbb{E}\left[\xi_{\infty} \cdot 1_{\eta_{L}<\infty}\right]+\mathbb{E}\left[\xi_{\infty} \cdot 1_{\eta_{L}=\infty}\right] \geq \mathbb{E}\left[\xi_{\infty} \cdot 1_{\eta_{L}<\infty}\right] \\
& \geq \frac{k}{(2(N-1))^{2}} \mathbb{P}\left(\eta_{L}<\infty\right)
\end{aligned}
$$

since on $\left\{\eta_{L}<\infty\right\}$ we have $\xi_{\infty}=W^{\prime}\left(\eta_{L}\right)$ and the largest coordinate of $W^{\prime}\left(\eta_{L}\right)$ is larger than $1 / 2$, implying that $\mu\left(W^{\prime}\left(\eta_{L}\right)\right) \geq \frac{1}{2(N-1)}$ and thus $h\left(W^{\prime}\left(\eta_{L}\right)\right)=F\left(W^{\prime}\left(\eta_{L}\right)\right)+$ $k \mu\left(W^{\prime}\left(\eta_{L}\right)\right)^{2} \geq \frac{k}{(2(N-1))^{2}}$. We also have

$$
\begin{gathered}
\xi_{0}=h\left(\mathcal{X}^{\prime}\left(\tau_{L}\right)\right)=F\left(\mathcal{X}^{\prime}\left(\tau_{L}\right)\right)+k \mu\left(\mathcal{X}^{\prime}\left(\tau_{L}\right)\right)^{2} \leq \frac{N-1}{L^{2}}+\frac{k}{L^{2}} \\
\Longrightarrow \mathbb{E} \xi_{0} \leq \frac{N+k-1}{L^{2}}
\end{gathered}
$$

since $\mathcal{X}^{\prime}\left(\tau_{L}\right) \subset[0,1 / L]$ and so $\mu\left(\mathcal{X}^{\prime}\left(\tau_{L}\right)\right) \in[0,1 / L]$.

Combining the above inequalities, we conclude that $\mathbb{P}\left(\eta_{L}<\infty\right) \rightarrow 0$ as $L \rightarrow \infty$. However, on $\eta_{L}=\infty$ the core of the regular $p$-contest process can be trivially coupled with the core of the borderless process $W^{\prime}(s)$ which converges to zero, so $\mathcal{X}^{\prime}(t) \rightarrow 0$ as well. Since $\mathbb{P}\left(\eta_{L}=\infty\right)$ can be made arbitrarily close to 1 by choosing a large $L$, we conclude that $\mathcal{X}^{\prime}(t) \rightarrow 0$ a.s.

\section{The Case $p>1$}

Throughout this section we suppose that $\zeta$ has a full support on $[0,1]$, and, unless explicitly stated otherwise, that $p>1$.

Theorem 2 (a) $\mathbb{P}\left(\left\{\mathcal{X}^{\prime}(t) \rightarrow 0\right\} \cup\left\{\mathcal{X}^{\prime}(t) \rightarrow 1\right\}\right)=1$;

(b) if $x_{(1)}(0) \geq 1 / p$ then $\mathbb{P}\left(\mathcal{X}^{\prime}(t) \rightarrow 1\right)=1$;

(c) if $x_{(k)}(0)>0$, where $k$ satisfies

$$
\{2 p(N-k)>N-2 p\} \Longleftrightarrow\left\{k<N-\frac{N}{2 p}+1\right\},
$$

then $\mathbb{P}\left(\mathcal{X}^{\prime}(t) \rightarrow 1\right)>0$.

Remark 2 In general, both convergences can have a positive probability. Let $N=3, p \in$ $(1,3 / 2)$, and

$$
\zeta= \begin{cases}U, & \text { with probability } 1 / 3 \\ 0, & \text { with probability } 1 / 3 \\ 1, & \text { with probability } 1 / 3\end{cases}
$$

where $U \in U[0,1]$ (so $\zeta$ has full support). Suppose we sample the points of $\mathcal{X}(0)$ from $\zeta$. If they all start off in 0 , then $p \mu \leq p / 3<1 / 2$, so they cannot escape from 0 . On the other hand, there is a positive probability they all start in $(1 / p, 1]$, and then Theorem 2(b) says that they converge to 1 . 
The key idea behind the proof of Theorem 2 is that one can actually find the "ruling" order statistic of the core; namely, there exists some non-random $k=k(N, p) \in\{1,2, \ldots, N-1\}$ such that $x_{(k)}(t) \rightarrow 0$ implies $\mathcal{X}^{\prime}(t) \stackrel{\text { a.s. }}{\longrightarrow} 0$, while $x_{(k)}(t) \nrightarrow 0$ implies that $\mathcal{X}^{\prime}(t) \stackrel{\text { a.s. }}{\longrightarrow} 1$.

We start with the following two results, which tells us that there is an absorbing area $\left[\frac{1}{p}, 1\right]$ for the process, such that, once the core enters this area, it will never leave it, and moreover the core will keep moving to the right.

Claim 1 Suppose that $x_{1} \leq x_{2} \leq x_{3} \leq \cdots \leq x_{N} \leq 1$ and $x_{2} \geq p^{-1}$. Then $\left\{x_{1}, \cdots, x_{N}\right\}^{\prime}=$ $\left\{x_{2}, \cdots, x_{N}\right\}$

Proof Let $\mu=\frac{x_{1}+\cdots x_{N}}{N}$. If $p \mu \geq x_{N}$ then the claim follows immediately; assume instead that $p \mu<x_{N}$. We need to check if $p \mu-x_{1}>x_{N}-p \mu$, that is, if

$$
2 p\left(x_{2}+\cdots+x_{N-1}\right)>(N-2 p)\left(x_{1}+x_{N}\right)
$$

However, since $x_{i} \geq x_{2}$ for $i=3, \ldots, N-1$ we have

$$
2 p\left(x_{2}+\cdots+x_{N-1}\right) \geq 2 p x_{2}(N-2) \geq 2(N-2)
$$

while $(N-2 p)\left(x_{1}+x_{N}\right) \leq 2(N-2 p)<2(N-2)$. Hence (3.2) follows.

Lemma 3 If $x_{(1)}\left(t_{0}\right) \geq 1 / p$ for some $t_{0}$, then $\mathcal{X}^{\prime}(t) \rightarrow 1$ a.s.

Proof If $x_{(1)}\left(t_{0}\right) \geq 1 / p$, then any point that lands in $[0,1 / p)$ is extreme, so $x_{(2)}(t) \geq 1 / p$ for all $t \geq t_{0}$. Choose any positive $\varepsilon<1-\frac{1}{p}$, and let $A_{t}=\left\{\zeta_{t+1}, \ldots, \zeta_{t+N-1} \in(1-\varepsilon, 1]\right\}$. Then if $A_{t}$ happens for $s>t_{0}$, any point in $[0,1-\varepsilon]$ is removed in preference to any of the new points coming in, so $x_{(2)}(s+N-1)>1-\varepsilon$. As a result, by Claim 1 we get that $\mathcal{X}^{\prime}(t) \in[0,1-\varepsilon]$ for all $t \geq s$.

On the other hand, $\mathbb{P}\left(A_{t}\right) \geq[f(\varepsilon)]^{N-1}>0$ (see (2.1)) for any $t$, and the events $A_{t}, A_{t+N}, A_{t+2 N}, \ldots$ are independent. Hence, eventually with probability 1 , one of the $A_{t}$ 's must happen for some $t>t_{0}$, so a.s. $\mathcal{X}^{\prime}(t) \in[0,1-\varepsilon]$ for all large $t$. Since $\varepsilon$ can be chosen arbitrary small, we get the result.

The next two results show that if the is some $\varepsilon>0$ such that infinitely often the core does not have any points in $[0, \varepsilon)$, then it must, in fact, converge to 1 .

Lemma 4 If $x_{(1)}\left(t_{0}\right) \geq \varepsilon$ for some $t_{0}$ and $\varepsilon>0$, then $\mathbb{P}\left(x_{(1)}\left(t_{0}+\ell\right) \geq p^{-1} \mid \mathcal{F}_{t}\right) \geq \delta$ for some $\ell=\ell(\varepsilon)$ and $\delta=\delta(\varepsilon)>0$.

Proof Suppose that for some $t$ we have $x_{(1)}(t) \geq \varepsilon$. We claim that it is possible to move $x_{(1)}$ to the right of $\frac{1+p}{2} \varepsilon$ in at most $N-1$ steps with positive probability, depending only on $p$ and $\varepsilon$. Indeed, if $x_{(1)}(t)>\frac{1+p}{2} \varepsilon$ then we are already done. Otherwise, if the new point $\zeta_{t+1}$ is sampled in $\left(\frac{1+p}{2} \varepsilon, p \varepsilon\right] \subset[0,1]$ it cannot be rejected. If at this stage $x_{(1)}(t+1)>\frac{1+p}{2} \varepsilon$, then we are done. If not, we proceed again by sampling $\zeta_{t+2} \in\left(\frac{1+p}{2} \varepsilon, p \varepsilon\right]$, etc. After at most $N-1$ steps of sampling new points in $\left(\frac{1+p}{2} \varepsilon, p \varepsilon\right]$, the leftmost point $x_{(1)}$ will have moved to the right of $\frac{1+p}{2} \varepsilon$.

Thus, in no more than $N-1$ steps, with probability no less than $\left[f\left(\frac{p-1}{2} \varepsilon\right)\right]^{N-1}>0, x_{(1)}$ is to the right of $\frac{1+p}{2} \varepsilon$. By iterating this argument at most $m$ times, where $m \in \mathbb{N}$ is chosen such that $\left[\frac{1+p}{2}\right]^{m} \varepsilon>1 / p$, we achieve that $x_{(1)}$ is to the right of $1 / p$ (for definiteness, one can chose $\ell=(N-1) m$ and $\delta=\left[f\left(\frac{p-1}{2} \varepsilon\right)\right]^{(N-1) m}$.) 
Lemma 5 Let $\varepsilon \in(0,1)$, and define $B(\varepsilon):=\left\{x_{(1)}(t) \geq \varepsilon\right.$ i.o. $\}$ Then $B(\varepsilon) \underset{\text { a.s. }}{\Longrightarrow}\left\{\mathcal{X}^{\prime}(t) \rightarrow 1\right\}$.

Corollary 1 We have $\left\{\liminf _{t \rightarrow \infty} x_{(1)}(t)>0\right\} \underset{\text { a.s. }}{\Longrightarrow}\left\{\mathcal{X}^{\prime}(t) \rightarrow 1\right\}$.

Proof of Lemma 5 Assume that $\varepsilon<\frac{1}{p}$ (otherwise the result immediately follows from Lemma 3). Also suppose that $\mathbb{P}(B(\varepsilon))>0$, since otherwise the result is trivial. Let $\ell$ and $\delta$ be the quantities from Lemma 4 .

Define

$$
\begin{aligned}
\tau_{0} & =\inf \left\{t>0: x_{(1)}(t)>\varepsilon\right\}, \\
\tau_{k} & =\inf \left\{t>\tau_{k-1}+\ell: x_{(1)}(t)>\varepsilon\right\}, \quad k \geq 1,
\end{aligned}
$$

with the convention that if $\tau_{k}=\infty$ then $\tau_{m}=\infty$ for all $m>k$. Notice that $B(\varepsilon)=$ $\bigcap_{k=0}^{\infty}\left\{\tau_{k}<\infty\right\}$. On $B(\varepsilon)$ we can also define $D_{\tau_{k}}=\left\{x_{(1)}\left(\tau_{k}+\ell\right) \geq 1 / p\right\}$. Since $\tau_{k}-\tau_{k-1}>\ell$ whenever both are finite, we have from Lemma 4 we have $\mathbb{P}\left(D_{\tau_{k+1}} \mid \mathcal{F}_{\tau_{k}}\right) \geq \delta$. Therefore,

$$
B(\varepsilon) \underset{\text { a.s. }}{\Longrightarrow}\left\{\sum_{k \geq 0} \mathbb{P}\left(D_{\tau_{k+1}} \mid \mathcal{F}_{\tau_{k}}\right)=\infty\right\}
$$

hence by Lévy's extension of the Borel-Cantelli lemma it follows that a.s. on $B(\varepsilon)$ ) infinitely many (and hence at least one) of $D_{\tau_{k}}$ occur, that is, $x_{(1)}\left(\tau_{k}+\ell\right) \geq 1 / p$. Now the result follows from Lemma 3.

Assume for now that $p<\frac{N}{2}$; in this case $N-\frac{N}{2 p}+1<N$ (see (3.1)). The case $p \geq \frac{N}{2}$ will be dealt with separately.

The following statement shows that if all the points to the right of $x_{(k)}$ lie very near each other, while the left-most one lies near zero, then it is to be removed.

Claim 2 Let $a \in(0,1]$ and suppose that $k \in\{2, \ldots, N-1\}$ satisfies (3.1). Then there exist small $\delta, \Delta>0$, depending on $N, k, p$, a such that if

$$
\begin{aligned}
0 & \leq x_{1} \leq \delta ; \\
x_{1} & \leq x_{i} \leq x_{N} \text { for } i=2, \ldots, N-1 ; \\
x_{k}, x_{k+1}, \ldots, x_{N} & \in[a(1-\Delta), a)
\end{aligned}
$$

then $\left\{x_{1}, \ldots, x_{N}\right\}^{\prime}=\left\{x_{2}, \ldots, x_{N}\right\}$.

Proof The condition to remove the leftmost point is $p \mu-\frac{x_{1}+x_{N}}{2}>0$ where $\mu=\left(x_{1}+\cdots+\right.$ $\left.x_{N}\right) / N$. However,

$$
\begin{aligned}
2 N\left(p \mu-\frac{x_{1}+x_{N}}{2}\right) & =2 p\left(x_{2}+\cdots+x_{N-1}\right)-(N-2 p) x_{1}-(N-2 p) x_{N} \\
& \geq 2 p\left(x_{k}+\cdots+x_{N-1}\right)-(N-2 p) \delta-(N-2 p) a \\
& \geq 2 p(N-k) a(1-\Delta)-(N-2 p) \delta-(N-2 p) a \\
& =a[2 p(N-k)(1-\Delta)-(N-2 p)]-(N-2 p) \delta
\end{aligned}
$$

The RHS is linear in $\delta$ and $\Delta$, and when $\delta=\Delta=0$ it is strictly positive by the assumption on $k$; hence it can also be made positive, by allowing $\delta>0$ and $\Delta>0$ to be sufficiently small. 
Corollary 2 Suppose that $\mathcal{X}(t)=\left\{x_{1}, \ldots, x_{N}\right\}$ satisfies the conditions of Claim 2 for some $a$ and $k$. Let $\delta$ be the quantity from this claim. Then

$$
\mathbb{P}\left(x_{(1)}(t+j)>\delta \text { for some } 1 \leq j \leq k \mid \mathcal{F}_{t}\right) \geq c=c_{a \Delta}>0 .
$$

Proof The probability to sample a new point $\zeta \in(a(1-\Delta), a]$ is bounded below by $f(a \Delta)$ where $f$ is the same function as in (2.1). On the other hand, if the new point is sampled in $(a(1-\Delta), a]$ then $\mathcal{X}(t+1)$ continues to satisfy the conditions of Claim 2 as long as the leftmost point is in $[0, \delta]$. By repeating this argument at most $k$ times and using the induction, we get the result with $c=[f(a \Delta)]^{k}>0$.

Lemma 6 Let $k \in \mathbb{N}$ satisfy (3.1). Then

$$
\left\{x_{(k)}(t) \nrightarrow 0\right\} \underset{\text { a.s. }}{\Longrightarrow}\left\{\mathcal{X}^{\prime}(t) \rightarrow 1\right\} .
$$

Proof Note that by Lemma 5, it suffices to show that $\left\{x_{(k)}(t) \nrightarrow 0\right\} \Longrightarrow\left\{x_{(1)}(t) \nrightarrow 00\right\}$.

If $x_{(k)}(t) \not \rightarrow 0$, there exists an $a>0$ such that $x_{(k)}(t) \geq a$ for infinitely many $t$ 's. Let $s$ be such a time. Now suppose that $\zeta_{s+i} \in I:=(a(1-\Delta), a]$ for $i=0,1, \ldots, N-1$ where $\Delta$ is defined in Claim 2; the probability of this event is strictly positive and depends only on $a$ and $\delta$ (see (2.1)). As long as there are points of $\mathcal{X}(s+i)$ on both sides of the interval $I$, none of the points inside $I$ can be removed; hence, for some $u \in\{s, s+1, \ldots, s+N-1\}$ we have that either $\min \mathcal{X}(u)>a(1-\Delta)$ or $\max \mathcal{X}(u) \leq a$. In the first case, $x_{(1)}(u)>a(1-\Delta)$.

In the latter case, both $x_{(N)}(u) \in I$ and $x_{(k)}(u) \in I$, since every time we replaced a point, the number of points to the left of $I$ did not increase (and there were initially at most $k-1$ of them). As a result

$$
a(1-\Delta) \leq x_{(k)}(u) \leq x_{(k+1)}(u) \leq \cdots \leq x_{(N)}(u) \leq a .
$$

Together with Corollary 2, this yields

$$
\left\{x_{(k)}(t) \geq a \text { i.o. }\right\} \underset{\text { a.s. }}{\Longrightarrow}\left\{x_{(1)}(t) \geq \min \{a(1-\Delta), \delta\} \text { i.o. }\right\} \underset{\text { a.s. }}{\Longrightarrow}\left\{x_{(1)}(t) \not \rightarrow 0\right\}
$$

which proves Lemma 6.

Claim 3 Let $A_{i}:=\left\{x_{(i)}(t) \rightarrow 0\right\}$ and suppose that for some $1 \leq k \leq N-2$ we have

$$
\{2 p(N-k-1)<N\} \Longleftrightarrow\left\{k>N-\frac{N}{2 p}-1\right\} .
$$

Then $A_{k} \subseteq\left\{\exists \lim _{t \rightarrow \infty} x_{(k+1)}(t)\right\}$.

Proof Fix any $a>0$. Let $\delta>0$ be so small that

$$
2 p N \delta<[N-2 p(N-k-1)] a .
$$

In the event $A_{k}$ there exists a finite $\tau=\tau_{\delta}(\omega)$ such that

$$
\left\{\sup _{t \geq \tau} x_{(k)}(t) \leq \delta\right\} \Longleftrightarrow\left\{\operatorname{card}\left(\mathcal{X}^{\prime}(t) \cap[0, \delta]\right) \geq k \text { for all } t \geq \tau .\right\}
$$

From now on assume that $t \geq \tau$. We will show below that $x_{(k+1)}(t+1) \leq \max \left\{x_{(k+1)}(t), a\right\}$.

To begin, let us prove that $x_{(k+1)}(t+1) \leq x_{(k+1)}(t)$ as long as $x_{(k+1)}(t)>\delta$. Indeed, if the new point $\zeta$ is sampled to the left of $x_{(k+1)}(t)$, then regardless of which point is to be removed, $x_{(k+1)}(t+1) \leq x_{(k+1)}(t)$. If the new point $\zeta$ is sampled to the right, then the farthest point from the $p$-centre of mass must be the rightmost one (and hence $x_{(k+1)}(t+1)=x_{(k+1)}(t)$ ) 
since there are exactly $k$ points in $[0, \delta]$ and none of these can be removed by the definition of $\tau$.

On the other hand, if $x_{(k+1)}(t) \leq \delta$ then either $x_{(k+2)}(t) \leq a$ or $x_{(k+2)}(t)>a$. In the first case, $x_{(k+1)}(t+1) \leq x_{(k+2)}(t) \leq a$ even if $x_{(1)}$ is removed. In the other case, when $x_{(k+2)}(t)>a$, we have $x_{(N-1)}>a$ as well, and

$$
\begin{aligned}
p \mu(\mathcal{X}(t+1)) & \leq p \frac{(k+1) \delta+(N-k-1) x_{(N)}}{N} \\
& <\frac{2 p N \delta-[N-2 p(N-k-1)] x_{(N)}+N x_{N}}{2 N} \\
& \leq \frac{\left.N x_{N}-\{[N-2 p(N-k-1)] a-2 p N \delta\}\right)}{2 N}<\frac{x_{(N)}}{2}
\end{aligned}
$$

by (3.4), so $x_{(N)}=x_{(N)}(t)$ must be removed and thus $x_{(k+1)}(t+1) \leq x_{(k+1)}(t)$.

Consequently, we obtained

$$
\begin{aligned}
A_{k} & \subseteq \bigcap_{t \geq \tau}\left\{x_{(k+1)}(t+1) \leq \max \left\{x_{(k+1)}(t), a\right\}\right\} \\
& \subseteq\left(\bigcup_{t \geq 0}\left\{x_{(k+1)}(s) \leq a \text { for all } s \geq t\right\}\right) \\
& \cup\left(\bigcup_{t \geq 0}\left\{x_{(k+1)}(s) \leq x_{(k+1)}(s+1) \text { for all } s \geq t\right\}\right) \\
& \subseteq\left\{\limsup _{t \rightarrow \infty} x_{(k+1)}(t) \leq a\right\} \cup\left\{\exists \lim _{t \rightarrow \infty} x_{(k+1)}(t)\right\}
\end{aligned}
$$

since $a>0$ is arbitrary, we get

$$
A_{k} \subseteq\left\{\limsup _{t \rightarrow \infty} x_{(k+1)}(t) \leq 0\right\} \cup\left\{\exists \lim _{t \rightarrow \infty} x_{(k+1)}(t)\right\}=\left\{\exists \lim _{t \rightarrow \infty} x_{(k+1)}(t) \geq 0\right\}
$$

Lemma 7 Suppose that (3.3) holds for some $1 \leq k \leq N-2$. Then $A_{k} \underset{\text { a.s. }}{\Longrightarrow} A_{k+1}$.

Proof Let $\tilde{A}_{k+1}^{\geq a}:=\left\{\lim _{t \rightarrow \infty} x_{(k+1)}(t) \geq a\right\}$ (the existence of this limit on $A_{k}$ follows from Claim 3). It suffices to show that $\mathbb{P}\left(A_{k} \cap \tilde{A}_{k+1}^{\geq a}\right)=0$ for all $a>0$; then from the continuity of probability we get that $\mathbb{P}\left(A_{k} \cap\left\{\lim _{t \rightarrow \infty} x_{(k+1)}(t)>0\right\}\right)=0$ and hence $A_{k} \underset{\text { a.s. }}{\Longrightarrow} A_{k+1}$.

Fix an $a>0$. Let

$$
C_{t}=\left\{x_{(k)}(t)<\frac{a}{3} \text { and } x_{(k+1)}(t)>\frac{2 a}{3}\right\}, \quad \bar{C}_{T}=\bigcap_{t \geq T} C_{t},
$$

then

$$
A_{k} \cap \tilde{A}_{k+1}^{\geq a} \subseteq \bigcup_{T \geq 0} \bar{C}_{T}=\left\{\exists T>0: x_{(k)}(t)<\frac{a}{3} \text { and } x_{(k+1)}(t)>\frac{2 a}{3} \text { for all } t \geq T\right\} .
$$

If the probability of the LHS is positive, then, using the continuity of probability and the fact that $\bar{C}_{T}$ is an increasing sequence of events, we obtain that $\lim _{T \rightarrow \infty} \mathbb{P}\left(\bar{C}_{T}\right)>0$. Consequently, there exists a non-random $T_{0}$ such that $\mathbb{P}\left(\bar{C}_{T_{0}}\right)>0$. 
This is, however, impossible, as at each time point $t$, with probability at least $f(a / 3)$ (see (2.1)) the new point $\zeta_{t}$ is sampled in $B:=\left(\frac{a}{3}, \frac{2 a}{3}\right)$ and then either $x_{(k)}(t+1) \in B$ or $x_{(k+1)}(t+1) \in B$. Formally, this means that

$$
\mathbb{P}\left(C_{t+1} \mid C_{t}, \mathcal{F}_{t}\right) \leq 1-f(a / 3) \text { for all } t \geq 0 .
$$

By induction, for all $k \geq 1$,

$$
\mathbb{P}\left(\bar{C}_{T_{0}} \mid \mathcal{F}_{T_{0}}\right) \leq \mathbb{P}\left(\bigcap_{T=T_{0}}^{T_{0}+k} C_{t} \mid \mathcal{F}_{T_{0}}\right) \leq[1-f(a / 3)]^{k} .
$$

Since $k$ is arbitrary, and $f(a / 3)>0$, by taking the expectation, we conclude that $\mathbb{P}\left(\bar{C}_{T_{0}}\right)=0$ yielding a contradiction.

Hence the probability of the event $A_{k} \cap \tilde{A}_{k+1}^{\geq a}$ is zero.

Corollary 3 Suppose that (3.3) holds for some $1 \leq k \leq N-2$. Then

$$
\left\{x_{(k)}(t) \rightarrow 0\right\} \underset{\text { a.s. }}{\Longrightarrow}\left\{\mathcal{X}^{\prime}(t) \rightarrow 0\right\} \text {. }
$$

Proof Observe that if $k$ satisfies (3.3) then $k+1$ satisfies (3.3) as well. Thus by iterating Lemma 7 we obtain that $A_{k} \underset{\text { a.s. }}{\Longrightarrow} A_{k+1} \underset{\text { a.s. }}{\Longrightarrow} A_{k+2} \underset{\text { a.s. }}{\Longrightarrow} \ldots \underset{\text { a.s. }}{\Longrightarrow} A_{N-1}$, i.e. $x_{(N-1)}(t) \rightarrow 0$, which is equivalent to the statement of Corollary.

Remark 3 Note that the condition (3.3) does not assume $p>1$; hence the conclusion of Corollary 3 holds for the case $0<p \leq 1$ as well.

For the case $p \geq \frac{N}{2}$ we have

Lemma 8 If $p \geq \frac{N}{2}$ then $\mathcal{X}^{\prime}(t) \rightarrow 1$ a.s.

Proof The case $p>\frac{N}{2}$ is easy: with a positive probability the newly sampled point $\zeta>0$ and then

$$
p \frac{x_{(1)}+\cdots+x_{(N-1)}+\zeta}{N}>\frac{x_{(1)}+\cdots+x_{(N-1)}+\zeta}{2} \geq \frac{x_{(1)}+\zeta}{2}
$$

hence it is the left-most point which is always removed, implying $\liminf _{t \rightarrow \infty} x_{(1)}(t)>0$. Hence by Corollary $1, \mathcal{X}^{\prime}(t) \rightarrow 1$ a.s.

For the case $p=\frac{N}{2}$ we notice that at each moment of time we either have a tie (between the left-most and right-most point) or remove the left-most point. However, we can only have a tie if $x_{(1)}(t)=\ldots=x_{(N-1)}(t)=0$; in this case, eventually the right-most point will be kept and the left-most removed. After this moment of time, there will be more ties, and the left-most point will always be removed, leading to the same conclusion as in the case $p>N / 2$.

Proof of Theorem 2 Part (b) follows from Lemma 3.

To prove part (c), note that unless $x_{(1)}(0)>0$ already, by repeating the arguments from the beginning of the proof of Lemma 6, with a positive probability we can "drag" the whole configuration in at most $N-1$ steps to the right of zero, that is, there is $0 \leq t_{0} \leq N-1$ such that $\mathbb{P}\left(\min \mathcal{X}^{\prime}\left(t_{0}\right)>0\right)>0$. Now we can apply Lemma 4 and then Lemma 3.

Let us now prove part (a). First, assume $p<\frac{N}{2}$. It is always possible to find an integer $k$ which satisfies both (3.1) and (3.3), so let $k$ be such that

$$
N-\frac{N}{2 p}-1<k<N-\frac{N}{2 p}+1
$$


(if $N /(2 p) \in \mathbb{N}$ this $k$ will be unique). Now the statement of the theorem follows from Corollary 3 and Lemma 6.

Finally, in case $p \geq \frac{N}{2}$ the theorem follows from Lemma 8 .

\section{Non-convergence to Zero for $p>1$ and $N=3$}

In this section we prove the following

Theorem 3 Suppose that $N=3, p>1$ and $\zeta$, restricted to some neighbourhood of zero, is a continuous random variable with a non-decreasing density (e.g. uniformly distributed). Then $\mathcal{X}^{\prime}(t) \rightarrow 1$ as $t \rightarrow \infty$ a.s.

Remark 4 - In case $p \geq 3 / 2$ we already know that $\mathcal{X}^{\prime}(t) \rightarrow 1$ for any initial configuration and any distribution (see Lemma 8), so we have to prove the theorem only for $p \in$ $(1,3 / 2)$.

- Simulations suggest that the statement of Theorem 3 holds, in fact, for a much more general class of distributions $\zeta$.

Let $\varepsilon \in(0,1 / 2)$ be such that $\zeta$ conditioned $^{2}$ on $\{\zeta \leq 2 \varepsilon\}$ has a non-decreasing density; according to the statement of the Theorem 3 such an $\varepsilon$ must exist. Let us fix this $\varepsilon$ from now on.

The idea of the proof will be based on finding a non-negative function $h:(0,1]^{2} \rightarrow \mathbb{R}_{+}$ which has the following three properties:

(i) $h(\cdot, \cdot)$ is non-increasing in each of its arguments;

(ii) $h\left(x_{(1)}(t), x_{(2)}(t)\right)$ is a supermartingale as long as $x_{(2)}(t) \leq \varepsilon$;

(iii) $h(\cdot, \cdot)$ goes to infinity when the first coordinate goes to zero.

From the supermartingale convergence theorem it would then follow that

$$
\mathbb{P}\left(\liminf _{t \rightarrow \infty} x_{(1)}(t)>0 \text { or } \limsup _{t \rightarrow \infty} x_{(2)}(t) \geq \varepsilon\right)=1 .
$$

Let us formally prepare for the proof of Theorem 3. As before, denote by $x_{1}, \ldots, x_{N}$ $N$ distinct points on $[0,1]$, and let $x_{(1)}, \ldots, x_{(N)}$ be this unordered $N$-tuple sorted in the increasing order. Let

$$
\left\{y_{1}, \ldots, y_{N-1}\right\}=\left\{x_{1}, \ldots, x_{N}\right\}_{p}^{\prime}
$$

be the unordered $N$-tuple $\left\{x_{1}, \ldots, x_{N}\right\}$ with the farthest point from $p$-centre of mass removed; w.l.o.g. assume that $y_{i}$ are already in the increasing order.

Lemma 9 The operation $\{\ldots\}_{p}^{\prime}$ is monotone in $p$, that is, if $\hat{p} \geq \tilde{p}$ and

$$
\begin{aligned}
& \left\{\hat{y}_{1}, \ldots, \hat{y}_{N-1}\right\}=\left\{x_{1}, \ldots, x_{N}\right\}_{\hat{p}}^{\prime}, \\
& \left\{\tilde{y}_{1}, \ldots, \tilde{y}_{N-1}\right\}=\left\{x_{1}, \ldots, x_{N}\right\}_{\tilde{p}}^{\prime}
\end{aligned}
$$

then $\hat{y}_{i} \geq \tilde{y}_{i}, i=1, \ldots, N-1$.

\footnotetext{
${ }^{2}$ Note that the full support assumption ensures that the probability of this event is positive.
} 
Proof Assume w.l.o.g. $x_{1} \leq \ldots \leq x_{N}$, and let $\mu=\mu\left(\left\{x_{1}, \ldots, x_{N}\right\}\right)$. Notice that, regardless of the value of $p$, the only points which can possibly be removed are $x_{1}$ or $x_{N}$ (since they are the two extreme points). Therefore, it suffices to show that $\left\{x_{1}, \ldots, x_{N}\right\}_{\tilde{p}}^{\prime}=\left\{x_{2}, \ldots, x_{N}\right\}$ implies $\left\{x_{1}, \ldots, x_{N}\right\}_{\hat{p}}^{\prime}=\left\{x_{2}, \ldots, x_{N}\right\}$. Note also that $\left|x_{1}-p \mu\right|=p \mu-x_{1}$ for all $p \geq 1$.

If $\tilde{p} \mu-x_{1}>\left|\tilde{p} \mu-x_{N}\right|$ and $\tilde{p} \mu-x_{N}>0$, that is, the $p$-centre of mass lies to the right of $x_{N}$, then $\hat{p} \mu>\tilde{p} \mu>x_{N}$ as well, and hence $x_{1}$ is discarded.

On the other hand, if $\tilde{p} \mu-x_{1}>\left|\tilde{p} \mu-x_{N}\right|$ and $\tilde{p} \mu<x_{N}$ then either $\hat{p} \mu<x_{N}$, or $\hat{p} \mu \geq x_{N}$. In the first case,

$$
\hat{p} \mu-x_{1}>\tilde{p} \mu-x_{1}>\left|\tilde{p} \mu-x_{N}\right|=x_{N}-\tilde{p} \mu>x_{N}-\hat{p} \mu=\left|x_{N}-\hat{p} \mu\right|
$$

so $x_{1}$ is discarded. In the second case, $p$-centre of mass lies to the right of $x_{N}$ and so $x_{1}$ is also discarded.

Lemma 10 Let $h$ be a real-valued function on the sets of $N$ real numbers. Suppose that $h$ is non-increasing in each of its arguments, namely

$$
h\left(x_{1}, x_{2}, \ldots, x_{i-1}, x_{i}^{\prime}, x_{i+1}, \ldots, x_{N}\right) \leq h\left(x_{1}, x_{2}, \ldots, x_{i-1}, x_{i}, x_{i+1}, \ldots, x_{N}\right)
$$

whenever $x_{i}^{\prime} \geq x_{i}$. Let $\mathcal{E}_{t}$ be some $\mathcal{F}_{t}$-measurable event, and suppose that

$$
\mathbb{E}\left(h\left(\mathcal{X}^{\prime}(t+1)\right) \mid \mathcal{F}_{t}\right) \leq h\left(\mathcal{X}^{\prime}(t)\right) \text { on } \mathcal{E}_{t}
$$

for $p=1$. Then (4.1) holds for $p>1$ as well.

\section{Proof Let}

$$
G_{p}\left(\mathcal{X}^{\prime}(t), \zeta_{t+1}\right)=\left\{x_{(1)}(t), x_{(2)}(t), \ldots, x_{(N-1)}(t), \zeta_{t+1}\right\}_{p}^{\prime}
$$

be the new core after the new point $\zeta_{t+1}$ is sampled and the farthest point from the $p$-centre of mass is removed; note that $\mathcal{X}^{\prime}(t+1)=G_{p}\left(\mathcal{X}^{\prime}(t), \zeta_{t+1}\right)$. Then on $\mathcal{E}_{t}$

$\mathbb{E}\left(h\left(\mathcal{X}^{\prime}(t+1)\right) \mid \mathcal{F}_{t}\right)=\mathbb{E}\left(h\left(G_{p}\left(\mathcal{X}^{\prime}(t), \zeta_{t+1}\right)\right) \mid \mathcal{F}_{t}\right) \leq \mathbb{E}\left(h\left(G_{1}\left(\mathcal{X}^{\prime}(t), \zeta_{t+1}\right)\right) \mid \mathcal{F}_{t}\right) \leq h\left(\mathcal{X}^{\prime}(t)\right)$

since the operation $\{\ldots\}_{p}^{\prime}$ is monotone in $p$ by Lemma 9 and $h$ is decreasing in each argument.

From now on assume $N=3$ and $p=1$. Denote $x_{(1)}(t)=a, x_{(2)}(t)=b$ and consider the events

$$
\begin{array}{ll}
L_{b}=\left\{\zeta_{t+1} \in\left((2 a-b)^{+}, a\right)\right\}, & R_{a}=\left\{\zeta_{t+1} \in(b, 2 b-a)\right\}, \\
B_{b}=\left\{\zeta_{t+1} \in\left(a, \frac{a+b}{2}\right)\right\}, & B_{a}=\left\{\zeta_{t+1} \in\left(\frac{a+b}{2}, b\right)\right\}
\end{array}
$$

(we assume that $b$ is smaller than $1 / 2$, yielding $2 b-a<1$.) If $x_{(2)}(t) \leq \varepsilon$ then $\mathcal{X}^{\prime}(t+1) \neq$ $\mathcal{X}^{\prime}(t)$ implies that one of the events $L_{b}, B_{b}, B_{a}$ or $R_{a}$ occurs (i.e. all points sampled outside of $\left((2 a-b)^{+}, 2 b-a\right)$ are rejected at time $\left.t+1\right)$. Let us study the core $\mathcal{X}^{\prime}(t+1)=\left\{\zeta_{t+1}, a, b\right\}^{\prime}$ on these events: on $L_{b}$ and $B_{b}$ we have $\mathcal{X}^{\prime}(t+1)=\{x, a\}$, while on $B_{a}$ and $R_{a}$ we have $\mathcal{X}^{\prime}(t+1)=\{x, b\}$.

We have, assuming $x_{(1)}(t)=a$ and $x_{(2)}(t)=b$,

$$
\mathbb{E}\left(h\left(\mathcal{X}^{\prime}(t+1)\right)-h\left(\mathcal{X}^{\prime}(t)\right) \mid \mathcal{F}_{t}\right)=\mathbb{E}\left(h\left(\{\zeta, a, b\}^{\prime}\right)-h(a, b)\right) .
$$


When $0 \leq a \leq b \leq \varepsilon$ we have $2 b-a \leq 2 \varepsilon$. Define

$$
g(x)=h\left(\{x, a, b\}^{\prime}\right)-h(a, b)= \begin{cases}h(x, a)-h(a, b), & \text { if } x \in\left((2 a-b)^{+}, a\right) ; \\ h(a, x)-h(a, b), & \text { if } x \in(a,(a+b) / 2) ; \\ h(x, b)-h(a, b), & \text { if } x \in((a+b) / 2, b) ; \\ h(b, x)-h(a, b), & \text { if } x \in(b, 2 b-a) \\ 0, & \text { otherwise, }\end{cases}
$$

which is positive in the first two cases, and negative in the next two. Let $\varphi(x)$ be the density of $\zeta$ conditioned on $\{\zeta \in[0,2 \varepsilon]\}$. By the monotonicity of $\varphi$ and the positivity (negativity resp.) of $g$ on the first (second resp.) interval,

$$
\begin{aligned}
\Delta(a, b): & =\mathbb{E}\left[g(\zeta) 1_{\zeta \in[0,2 \varepsilon]}\right]=\int_{(2 a-b)^{+}}^{\frac{a+b}{2}} g(x) \varphi(x) \mathrm{d} x+\int_{\frac{a+b}{2}}^{2 b-a} g(x) \varphi(x) \mathrm{d} x \\
\leq & \varphi\left(\frac{a+b}{2}\right) \int_{(2 a-b)^{+}}^{\frac{a+b}{2}} g(x) \mathrm{d} x \\
& +\varphi\left(\frac{a+b}{2}\right) \int_{\frac{a+b}{2}}^{2 b-a} g(x) \mathrm{d} x=\varphi\left(\frac{a+b}{2}\right) \cdot \Lambda,
\end{aligned}
$$

where

$$
\begin{aligned}
\Lambda= & \Lambda(a, b)=\int_{(2 a-b)^{+}}^{a}(h(x, a)-h(a, b)) \mathrm{d} x+\int_{a}^{\frac{a+b}{2}}(h(a, x)-h(a, b)) \mathrm{d} x \\
& +\int_{\frac{a+b}{2}}^{b}(h(x, b)-h(a, b)) \mathrm{d} x+\int_{b}^{2 b-a}(h(b, x)-h(a, b)) \mathrm{d} x .
\end{aligned}
$$

So if we can establish that $\Lambda \leq 0$ for a suitable function $h$, then indeed $\Delta(a, b) \leq 0$, and the supermartingale property follows.

Remark 5 Notice that the method of proof, presented here, could possibly work for $N>3$ as well; that is, if one can find a function $h\left(x_{1}, \ldots, x_{N-1}\right)$, which is positive and decreasing in each of its arguments, and $h\left(\mathcal{X}^{\prime}(t)\right)$ is a supermartingale provided $\max \mathcal{X}^{\prime}(t)<\varepsilon$ for some $\varepsilon>0$. Unfortunately, however, we were not able to find such a function.

Set

$$
h(x, y)=-2 \log \left(\max \left\{x, \frac{y}{2}\right\}\right) \geq 0 ;
$$

it is easy to check $h$ is indeed monotone in each of its arguments as long as $x, y \in(0,1]$. Let us now compute the integrals in the expression for $\Lambda$. We have

$$
\Lambda= \begin{cases}3(a-b) \ln 2-3 a+2 b, & \text { if } a \leq \frac{b}{3} \\ (a+b) \ln (a+b)-(a+b) \ln a+(a-5 b) \ln 2+b, & \text { if } \frac{b}{3}<a \leq \frac{b}{2} \\ (a+b) \ln (a+b)+(2 a-4 b) \ln b+3(b-a) \ln a+(b-5 a) \ln 2+b, & \text { if } \frac{b}{2}<a \leq \frac{2 b}{3} \\ (a+b) \ln (a+b)+(2 a-4 b) \ln b+(5 b-7 a) \ln a-(a+b) \ln 2 & \\ \quad+3(b-a)+(4 a-2 b) \ln (2 a-b), & \text { if } \frac{2 b}{3}<a \leq b .\end{cases}
$$

It turns out that $h\left(\mathcal{X}^{\prime}(t)\right)$ indeed has a non-positive drift, provided $0<a \leq b \leq \varepsilon$, as is shown by the following 
Lemma $11 \Lambda \leq 0$ for $a, b \in(0,1 / 2]$.

Proof Substitute $a=b v$ in the expression for $\Lambda$. Then for $v \leq 1 / 3$ we easily obtain $\Lambda=-b[3 v(1-\ln 2)+\ln 8-2] \leq 0$.

For $1 / 3<v \leq 1 / 2$ we have $2 \Lambda=-b C_{1}(v) \leq 0$ where

$$
C_{1}(v)=(1+v) \ln \frac{v}{1+v}+(5-v) \ln 2-1>0,
$$

since $\frac{\partial^{2} C_{1}(v)}{\partial^{2} v}=-\frac{1}{v^{2}(1+v)}<0$ and hence $\min _{1 / 3 \leq v \leq 1 / 2} C_{1}(v)$ is achieved at one of the endpoints $v=1 / 3$ or $v=1 / 2$; the values there are $C_{1}(1 / 3)=\ln (4)-1>0$ and $C(1 / 2)=$ $\frac{1}{2} \ln \left(\frac{512}{27}\right)-1>0$ respectively.

For $1 / 2<v \leq 2 / 3$ we have $\Lambda=-b C_{2}(v) \leq 0$ where

$$
C_{2}(v)=-(1+v) \ln (1+v)+(3 v-3) \ln v-1+(5 v-1) \ln 2>0,
$$

since $\frac{\partial^{2} C_{2}(v)}{\partial^{2} v}=\frac{2 v^{2}+6 v+3}{v^{2}(1+v)}>0$ and $\left.\frac{\partial C_{2}(v)}{\partial v}\right|_{\nu=2 / 3}=\ln \left(\frac{256}{45}\right)-\frac{5}{2}<0$ implies that $\frac{\partial C_{2}(v)}{\partial v}<0$ for all $v \in[1 / 2,2 / 3]$ and hence $\min _{1 / 2 \leq v \leq 2 / 3} C_{2}(v)=C_{2}(2 / 3)=\frac{1}{3} \ln \left(\frac{104976}{3125}\right)-1>0$.

Finally, for $2 / 3<v \leq 1$ we have $\Lambda=-b C_{3}(v) \leq 0$, where

$$
C_{3}(v)=v \log \frac{2 v^{7}}{(2 v-1)^{4}(v+1)}+\log \frac{2(2 v-1)^{2}}{v^{5}(v+1)}+3(v-1)>0
$$

since

$$
\frac{\mathrm{d}^{2} C_{3}(v)}{\mathrm{d} v^{2}}=\frac{(2 v+5)\left(2 v^{2}-1\right)}{\left.(2 v-1) v^{2}(v+1)\right)}
$$

changes its sign from - to + at $1 / \sqrt{2} \in(2 / 3,1)$ and therefore $\frac{\partial C_{3}(v)}{\partial v}$ achieves its maximum at the endpoints of the interval; thus

$$
\max _{2 / 3 \leq v \leq 1} \frac{\partial C_{3}(v)}{\partial v}=\max _{\nu=2 / 3,1} \frac{\partial C_{3}(v)}{\partial v}=\max \left\{-\frac{5}{2}+\ln \left(\frac{256}{45}\right), 0\right\}=0
$$

Therefore, $C_{3}(v)$ is decreasing and hence $\min _{2 / 3 \leq \nu \leq 1} C_{3}(v)=C_{3}(1)=0$.

Proof of Theorem 3 We will show that $\mathbb{P}\left(\mathcal{X}^{\prime}(t) \rightarrow 0\right)=0$, which will imply by Theorem 2(a) that $\mathbb{P}\left(\mathcal{X}^{\prime}(t) \rightarrow 1\right)=1$; we shall do it by showing that

$$
0 \leq \mathbb{P}\left(\mathcal{X}^{\prime}(t) \rightarrow 0\right) \leq \mathbb{P}\left(\left\{\liminf _{t \rightarrow \infty} x_{(1)}(t)=0\right\} \bigcap\left\{\limsup _{t \rightarrow \infty} x_{(2)}(t)<\varepsilon\right\}\right)=0 .
$$

Indeed, fix some $\varepsilon \in(0,1 / 2)$ and let $\tau_{0}=0$. For $\ell=1,2, \ldots$, define the sequence of stopping times

$$
\begin{aligned}
\eta_{\ell} & =\inf \left\{t>\tau_{\ell-1}: x_{(2)}(t) \leq \varepsilon\right\}, \\
\tau_{\ell} & =\inf \left\{t>\eta_{\ell}: x_{(2)}(t)>\varepsilon\right\},
\end{aligned}
$$

so that $\tau_{0}<\eta_{1}<\tau_{1}<\eta_{2}<\tau_{2}<\ldots$ with the conventions that if one of the stopping times is infinite, so is the rest of them. Define also $\ell^{*}=\inf \left\{\ell \geq 1: \tau_{\ell}=+\infty\right\}$.

If $\ell^{*}=\infty$, that is, $\tau_{\ell}<\infty$ for all $\ell$, we immediately have $\lim \sup _{t \rightarrow \infty} x_{(2)}(t) \geq \varepsilon$ and we are done; so assume that for some $\ell^{*} \geq 1$ we have $\tau_{\ell^{*}-1}<\infty=\tau_{\ell^{*}}$. If $\eta_{\ell^{*}}=\infty$, then $x_{(2)}(t)>\varepsilon$ for all $t \geq \tau_{\ell^{*}-1}$ and thus again $\limsup _{t \rightarrow \infty} x_{(2)}(t) \geq \varepsilon$; hence $\ell^{*}<\infty$ and $\eta_{\ell^{*}}<\infty$ on the event $\left\{\lim \sup _{t \rightarrow \infty} x_{(2)}(t)<\varepsilon\right\}$. 
On the other hand, as long as $\eta_{\ell}<\infty$, we can define

$$
\xi_{s}^{(\ell)}=h\left(\mathcal{X}^{\prime}\left(\eta_{\ell}+s\right)\right), \quad s \geq 0 \quad \text { and } \tilde{\xi}_{s}^{(\ell)}=\xi_{\min \left\{s, \tau_{\ell}-\eta_{\ell}\right\}}^{(\ell)} .
$$

where $h$ is given by (4.2).

By Lemmas 10 and 11 we have

$$
\mathbb{E}\left(\left[h\left(\mathcal{X}^{\prime}(t+1)\right)-h\left(\mathcal{X}^{\prime}(t)\right)\right] 1_{x_{(2)}(t) \leq \varepsilon} \mid \mathcal{F}_{t}\right) \leq 0,
$$

hence $\tilde{\xi}_{s}^{(\ell)}$, the process $\xi^{(\ell)}$ stopped at the time when $x_{(2)}$ exceeds $\varepsilon$, is a non-negative supermartingale, hence it must converge to a finite value. In case $\tau_{\ell}=+\infty$ this means that $\liminf _{t \rightarrow \infty} x_{(1)}(t)>0$ since the function $h(a, b)$ goes to infinity when $a \downarrow 0$. Thus we have established (4.3).

Acknowledgements Open access funding provided by Lund University. We would like to thank the anonymous referees for very careful reading our manuscript and for giving many useful suggestions and corrections.

Open Access This article is licensed under a Creative Commons Attribution 4.0 International License, which permits use, sharing, adaptation, distribution and reproduction in any medium or format, as long as you give appropriate credit to the original author(s) and the source, provide a link to the Creative Commons licence, and indicate if changes were made. The images or other third party material in this article are included in the article's Creative Commons licence, unless indicated otherwise in a credit line to the material. If material is not included in the article's Creative Commons licence and your intended use is not permitted by statutory regulation or exceeds the permitted use, you will need to obtain permission directly from the copyright holder. To view a copy of this licence, visit http://creativecommons.org/licenses/by/4.0/.

\section{Appendix: The Calculations for the Proof of Lemma 2.}

Observe that all expressions for $\mathbf{A}_{j}$ are fractions of the polynomials in $(a, f, p, M)$; moreover, their denominators

$$
\begin{array}{ll}
3 M(M-1) & \left(\text { for } \mathbf{A}_{1}\right), \\
3 M(M-1)(M+1-2 p)^{3} & \left(\text { for } \mathbf{A}_{2} \text { and } \mathbf{A}_{4}\right) \\
12 M(M-1)(M+1-2 p)^{3} p^{3} & \left(\text { for } \mathbf{A}_{3} \text { and } \mathbf{A}_{5}\right)
\end{array}
$$

are always positive. Throughout the rest of the proof let $n(w)$ denote the numerator of such a fraction $w$.

\section{Case 1: $I_{1} \leq 0$}

Observe that

$$
n\left(\mathbf{A}_{1}\right)=-2 M^{2}-3 M \mu+2 M+1+[3 M \mu-1] M p
$$

and the term in the square brackets is positive as $M \mu \geq 1$, so the maximum of $n\left(\mathbf{A}_{1}\right)$ is achieved at the highest possible value of $p$. However, in this case we have $p \leq p_{1}$, hence

$$
n\left(\mathbf{A}_{1}\right) 1_{X_{1} \leq 0} \leq\left. n\left(\mathbf{A}_{1}\right)\right|_{p=p_{1}}=-\frac{s_{1}}{2 \mu}
$$

where

$$
s_{1}=\left(M^{2}-2\right) \mu+(1-6 \mu)(1-\mu) M+1
$$




$$
=\left\{\begin{array}{l}
3(2 \mu-1)^{2}, \text { if } M=2 ; \\
4 \mu^{2}+1 / 2+14(\mu-1 / 2)^{2}, \text { if } M=3 ; \\
(M-3)\left[(M-4) \mu+6 \mu^{2}+1\right]+\left.s_{1}\right|_{M=3}, \text { if } M \geq 4
\end{array}\right.
$$

Hence $s_{1} \geq 0$ for $M=2,3, \ldots$ and thus $\mathbf{I}_{1} \leq 0$.

\section{Case $2: I_{2} \leq 0$}

Here

$$
n\left(\mathbf{A}_{2}\right)=-4[a(M-p+1)-M \mu p]^{2} s_{2}
$$

where

$$
\begin{aligned}
s_{2}= & M^{3} \mu p-4 M^{2} \mu p^{2}-M^{3} a+2 M^{2} a p+5 M^{2} \mu p \\
& +2 M a p^{2}-3 M^{2} \mu-6 M a * p+4 M \mu p \\
& +3 M a-3 M \mu-2 a p+2 a,
\end{aligned}
$$

and we need to show that $s_{2} \geq 0$.

Assume first $M=2$. Then (using the fact that $\mu=(1+a) / 2$ )

$$
X_{2} \geq 0 \Longleftrightarrow p \geq \frac{3 a+3}{4 a+2} \geq 1
$$

which is impossible; so from now on $M \geq 3$.

To establish $\mathbf{I}_{2} \leq 0$, it will suffice to demonstrate that

$$
s_{3}:=2 M s_{2}-2 M^{3}(M \mu+a) X_{2} \geq 0
$$

as $\mathbf{I}_{2}$ has a factor $1_{X_{2} \geq 0}$, and $s_{2} 1_{X_{2} \geq 0} \geq \frac{s_{3}}{2 M} 1_{X_{2} \geq 0}$. Substituting

$$
p=\left[\frac{1}{2}+\frac{1}{2 M}\right]+\left[\frac{1}{2}-\frac{1}{2 M}\right] w
$$

where $w \in[0,1)$ corresponding to the condition (2.4), we get

$$
\begin{aligned}
s_{3}= & M\left(-2 M^{2} \mu w^{2}+M^{2} \mu w+4 M \mu w^{2}+M^{3}\right. \\
& \left.-3 M^{2} \mu-M \mu w-2 \mu w^{2}+M^{2}-M \mu+2 \mu\right) \\
& -a \cdot(M-1)\left[M\left((M-1)^{2}-(w-2)^{2}\right)+(1-w)\left(M^{2}-w-1\right)\right]
\end{aligned}
$$

The expression in the square brakets is non-negative for $M \geq 3$, so the minimum of $s_{3}$ is achieved when $a=1$; i.e.

$$
\begin{aligned}
s_{3} \geq\left. s_{3}\right|_{a=1}= & -2 M^{3} \mu w^{2}+M^{3} \mu w+4 M^{2} \mu w^{2} \\
& -3 M^{3} \mu+M^{3} w-M^{2} \mu w+M^{2} w^{2} \\
& -2 M \mu w^{2}+3 M^{3}-M^{2} \mu-5 M^{2} w-2 M w^{2} \\
& +2 M^{2}+2 M \mu+4 M w+w^{2}-2 M-1=: s_{4}
\end{aligned}
$$

But

$$
\frac{\partial s_{4}}{\partial \mu}=-M\left((3-w) M^{2}+(1+w) M-2+2(M-1)^{2} w^{2}\right)<0
$$


so

$$
s_{4} \geq\left. s_{4}\right|_{\mu=1}=(1-w)(M-1)(w M(2 M-3)+M+w+1) \geq 0 .
$$

\section{Case 3: $I_{3} \leq 0$}

Here

$$
n\left(\mathbf{A}_{3}\right)=-(M+1)(1-a) s_{5}
$$

and it suffices to show that $s_{5} \geq 0$. If $M=2$, then $\mu=(a+1) / 2$ and $p \geq 3 / 4$, so

$$
\begin{aligned}
s_{5}= & 3(3-2 p)\left[(1-a)^{2}(8 p-5)+\left(32(1-a)^{2}+144 a\right)(1-p)^{4}\right. \\
& \left.+12(1-p)^{2}(4 p+a(4 a p+10 p-3))\right] \geq 0 .
\end{aligned}
$$

For $M \geq 3$, let $M=3+\delta, \delta=0,1, \ldots$ Then $s_{5}=\sum_{i=0}^{5} e_{i+1} \delta^{i}$ where we will show that all $e_{i} \geq 0$. Indeed, we have

$$
\begin{aligned}
e_{1}= & -432 a \mu p^{5}-1296 \mu^{2} p^{5}+288 a^{2} p^{4}+2736 a \mu p^{4} \\
& -144 a p^{5}+432 p^{4} \mu^{2}-432 \mu p^{5}-1632 p^{3} a^{2} \\
& -2880 a \mu p^{3}+1200 a p^{4}+4320 \mu^{2} p^{3}+2736 \mu p^{4} \\
& +2624 p^{2} a^{2}-1152 a \mu p^{2}-3744 a p^{3}-1728 \mu^{2} p^{2} \\
& -2880 \mu p^{3}+288 p^{4}-1536 p a^{2}+4928 p^{2} a-1152 p^{2} \mu \\
& -1632 p^{3}+768 a^{2}-3072 a p+2624 p^{2} \\
& +1536 a-1536 p+768 \\
e_{2}= & -288 a \mu p^{5}-1296 \mu^{2} p^{5}+168 a^{2} p^{4}+2208 a \mu p^{4}-48 a p^{5} \\
& -360 p^{4} \mu^{2}-288 \mu p^{5}-1160 p^{3} a^{2} \\
& -1392 a \mu p^{3}+600 a p^{4}+6984 \mu^{2} p^{3}+2208 \mu p^{4} \\
& +1760 p^{2} a^{2}-3840 a \mu p^{2}-2264 a p^{3}-2016 \mu^{2} p^{2} \\
& -1392 \mu p^{3}+168 p^{4}-576 p a^{2}+2720 p^{2} a-3840 p^{2} \mu \\
& -1160 p^{3}+768 a^{2}-1152 a p+1760 p^{2} \\
& +1536 a-576 p+768 \\
e_{3}= & -48 a \mu p^{5}-432 \mu^{2} p^{5}+24 a^{2} p^{4}+576 a \mu p^{4}-600 p^{4} \mu^{2} \\
& -48 \mu p^{5}-268 p^{3} a^{2}+216 a \mu p^{3}+72 a p^{4} \\
& +4404 \mu^{2} p^{3}+576 \mu p^{4}+324 p^{2} a^{2}-3240 a \mu p^{2} \\
& -412 a p^{3}-876 \mu^{2} p^{2}+216 \mu p^{3}+24 p^{4}+336 p a^{2} \\
& +180 p^{2} a-3240 p^{2} \mu-268 p^{3}+288 a^{2}+672 a p \\
& +324 p^{2}+576 a+336 p+288 \\
e_{4}= & -48 \mu^{2} p^{5}+48 a \mu p^{4}-216 p^{4} \mu^{2}-20 p^{3} a^{2}+192 a \mu p^{3} \\
& +1356 \mu^{2} p^{3}+48 \mu p^{4}-4 p^{2} a^{2}-1164 a \mu p^{2} \\
& -20 a p^{3}-168 \mu^{2} p^{2}+192 \mu p^{3}+228 p a^{2}-112 p^{2} a \\
& +230
\end{aligned}
$$




$$
\begin{aligned}
& -1164 p^{2} \mu-20 p^{3}+48 a^{2}+456 a p-4 p^{2} \\
& +96 a+228 p+48 \\
e_{5}= & -24 p^{4} \mu^{2}+24 a \mu p^{3}+204 \mu^{2} p^{3}-4 p^{2} a^{2}-192 a \mu p^{2} \\
& -12 \mu^{2} p^{2}+24 \mu p^{3}+45 p a^{2}-16 p^{2} a \\
& -192 p^{2} \mu+3 a^{2}+90 a p-4 p^{2}+6 a+45 p+3 \\
e_{6}= & 360 p(a+1-2 \mu p)^{2} \geq 0 .
\end{aligned}
$$

The fact that $e_{6} \geq 0$ is trivial; we will prove separately that $e_{1}, \ldots, e_{5} \geq 0$ below. In what follows, we substitute $p=\frac{1+v}{2}$, where $v \in(0,1)$.

\section{Proof that $e_{1} \geq 0$}

We have

$$
\frac{\partial^{2} e_{1}}{\partial a^{2}}=4\left[9 v^{4}-66 v^{3}+76 v^{2}+2 v+235\right]>0,
$$

hence $e_{1}$ achieves its minimum at

$$
a_{c r}=\frac{9 v^{5}-105 v^{4}+426 v^{3}-46 n u^{2}+397 v-1669+9 \mu(1+v)^{2}\left(3 v^{3}-29 v^{2}+13 v+109\right)}{8\left[9 v^{4}-66 v^{3}+76 v^{2}+2 v+235\right]}
$$

which solves $\frac{\partial e_{1}}{\partial a}=0$. Note that it is possible that $a_{c r} \notin[0,1]$. However, in any case,

$$
e_{1} \geq\left. e_{1}\right|_{a=a_{c r}}=\frac{1}{32} \cdot \frac{3(1+v)^{2} c_{1}}{9 v^{4}-66 v^{3}+76 v^{2}+2 v+235}
$$

so it will suffice to show that

$$
\begin{aligned}
c_{1}= & 16(1-v)^{2} c_{1 a}+3(1-\mu) c_{1 b}, \text { where } \\
c_{1 a}= & -27 v^{6}+144 v^{5}-102 v^{4}+1620 v^{3}-9883 v^{2}+12484 v+1732 \\
c_{1 b}= & 81 \mu v^{8}-108 \mu v^{7}+135 v^{8}-1260 \mu v^{6} \\
& -828 v^{7}-12276 \mu v^{5}+276 v^{6}+84774 \mu v^{4} \\
& -4404 v^{5}-157140 \mu v^{3}+69170 v^{4}+152628 \mu v^{2} \\
& -198372 v^{3}-156108 \mu v+182084 v^{2} \\
& +27969 \mu-60588 v+73967
\end{aligned}
$$

is positive. We have

$$
c_{1 a}=3 v^{3}\left(540-9 v^{3}+48 v^{2}-34 v\right)+v(12484-9883 v)+1732>0 .
$$

Similarly,

$$
c_{1 b}=61440(1-\mu)+(1-v)\left[c_{1 b 1}+c_{1 b 2} \mu\right]
$$

where

$$
\begin{aligned}
c_{1 b 1} & =\left(-135 v^{7}+693 v^{6}+417 v^{5}+4821 v^{4}\right)-64349 v^{3}+134023 v^{2}-48061 v+12527 \\
& \geq-64349 v^{3}+134023 v^{2}-48061 v+12527 \geq 1000\left(-67 v^{3}+134 v^{2}-67 v+12\right)
\end{aligned}
$$




$$
=\frac{1000}{27}\left[56+67(4-3 v)(1-3 v)^{2}\right]>0
$$

and

$$
\begin{aligned}
c_{1 b 2} & =\left(-81 v^{7}+27 v^{6}+1287 v^{5}+13563 v^{4}\right)-71211 v^{3}+85929 v^{2}-66699 v+894 \\
& \geq-71211 v^{3}+85929 v^{2}-66699 v+89409>80000\left(-v^{3}+v^{2}-v+1\right) \geq 0 .
\end{aligned}
$$

So, $c_{1 b 1}, c_{1 b 2}>0 \Longrightarrow c_{1 b}>0$ and since $c_{1 a}>0$ we have $c_{1} \geq 0$ and thus $e_{1} \geq 0$.

\section{Proof that $e_{2} \geq 0$}

We have

$$
\frac{\partial^{2} e_{2}}{\partial a^{2}}=21 v^{4}-206 v^{3}+136 v^{2}+398 v+1571>0
$$

so, similarly to the previous case,

$$
e_{2} \geq\left. e_{2}\right|_{a=a_{c r}}=\frac{3\left(1+v^{2}\right)\left[582912(1-\mu)^{2}+(1-v) c_{2}\right]}{8\left[21 v^{4}-206 v^{3}+136 v^{2}+398 v+1571\right]}
$$

where

$$
a_{c r}=\frac{3 v^{5}-60 v^{4}+296 v^{3}-82 v^{2}-155 v-2786+\left(18 v^{3}-222 v^{2}-150 v+2010\right)(1+v)^{2} \mu}{2\left[21 v^{4}-206 v^{3}+136 v^{2}+398 v+1571\right]}
$$

solves $\frac{\partial e_{2}}{\partial a}=0$ and

$$
\begin{aligned}
c_{2}= & 3 v^{7}-123 v^{6}+1330 v^{5}-1918 v^{4}-28897 v^{3}+65177 v^{2}+93100 v+120544 \\
& +\left(36 v^{7}-624 v^{6}+348 v^{5}+25616 v^{4}-7332 v^{3}-272368 v^{2}-134556 v+688784\right) \\
& +\left(108 v^{7}-72 v^{6}-4848 v^{5}-35916 v^{4}+247548 v^{3}-252720 v^{2}+144456 v-647676\right) \mu^{2}
\end{aligned}
$$

Now,

$$
\begin{aligned}
\frac{\partial^{2} c_{2}}{\partial \mu^{2}}= & v^{4}\left(216 v^{3}-144 v^{2}-9696 v-71832\right) \\
& +v^{3}(495096 v-505440)+(288912 v-1295352)<0
\end{aligned}
$$

hence the minimum of $c_{2}$ w.r.t. $\mu \in[0,1]$ can be achieved either at $\mu=0$ or at $\mu=1$. At the same time

$$
\begin{aligned}
\left.c_{2}\right|_{\mu=0}= & 3 v^{7}+1330 v^{5}+65177 v^{2}+93100 v \\
& +\left(120544-123 v^{6}-1918 v^{4}-28897 v^{3}\right)>0, \\
\left.c_{2}\right|_{\mu=1}= & (1-v)\left(161652-147 v^{6}+672 v^{5}+3842 v^{4}\right. \\
& \left.+16060 v^{3}+(264652-195259 v) v\right) \geq 0,
\end{aligned}
$$

so $c_{2} \geq 0$ and hence $e_{2} \geq 0$. 


\section{Proof that $e_{3} \geq 0$}

We have

$$
\frac{\partial^{2} e_{3}}{\partial a^{2}}=3 v^{4}-55 v^{3}-21 v^{2}+471 v+1010>0
$$

so, similarly to the previous case,

$$
e_{3} \geq\left. e_{3}\right|_{a=a_{c r}}=\frac{3(1+v)^{2}\left[(1-v)^{2} c_{3 a}+(1-\mu) c_{3 b}\right]}{8\left(3 v^{4}-55 v^{3}-21 v^{2}+471 v+1010\right)}
$$

where

$$
a_{c r}=\frac{-9 v^{4}+67 v^{3}+165 v^{2}-579 v-1820+3(1+v)^{2} \mu\left(v^{3}-21 v^{2}-63 v+499\right)}{2\left(3 v^{4}-55 v^{3}-21 v^{2}+471 v+1010\right)}
$$

solves $\frac{\partial e_{3}}{\partial a}=0$ and

$$
\begin{aligned}
c_{3 a}= & -3 v^{6}+12 v^{5}+632 v^{4}+1794 v^{3}-37624 v^{2}+65244 v+64877>0 \\
c_{3 b}= & 2(1-v)\left(-3 v^{7}+12 v^{6}+652 v^{5}+2417 v^{4}\right. \\
& \left.-42561 v^{3}+73864 v^{2}+41336 v+91323\right) \\
& +(1-\mu)\left(3 v^{6}\left(220-v^{2}+4 v\right)\right. \\
& \left.+2 v\left(1490 v^{4}-22993 v^{3}+39898 v^{2}+890 v+109262\right)+8477\right) \geq 0
\end{aligned}
$$

Hence $e_{3} \geq 0$.

\section{Proof that $e_{4} \geq 0$}

We have

$$
\frac{\partial^{2} e_{4}}{\partial a^{2}}=209 v+317-5 v^{3}-17 v^{2}>0
$$

so, similarly to the previous case,

$$
e_{4} \geq\left. e_{4}\right|_{a=a_{c r}}=\frac{3(1+v)^{2}\left[(1-v)^{2} c_{4 a}+4(1-\mu) c_{4 b}\right]}{8\left(209 v+317-5 v^{3}-17 v^{2}\right)}
$$

where

$$
a_{c r}=\frac{5 v^{3}+71 v^{2}-329 v-587+6 \mu(1+v)^{2}\left(88-10 v-v^{2}\right)}{2\left(209 v+317-5 v^{3}-17 v^{2}\right)}
$$

solves $\frac{\partial e_{4}}{\partial a}=0$ and

$$
\begin{aligned}
c_{4 a}= & 8 v^{4}+40 v^{3}-1395 v^{2}+4354 v+4757>0 \\
c_{4 b}= & 4(1-v)\left(4 v^{5}+21 v^{4}-712 v^{3}+2011 v^{2}+3102 v+3050\right) \\
& +(1-\mu)\left(2 v^{6}+11 v^{5}-360 v^{4}+912 v^{3}+1705 v^{2}+3655 v+543\right) \geq 0 .
\end{aligned}
$$

Hence $e_{4} \geq 0$. 


\section{Proof that $e_{5} \geq 0$}

We have

$$
\frac{\partial^{2} e_{5}}{\partial a^{2}}=49+41 v-2 v^{2}>0
$$

so, similarly to the previous case,

$$
e_{5} \geq\left. e_{5}\right|_{a=a_{c r}}=\frac{3(1+v)^{2}\left[(1-v)^{2} c_{5 a}+(1-\mu) c_{5 b}\right]}{2\left(49+41 v-2 v^{2}\right)}
$$

where

$$
a_{c r}=\frac{4 v^{2}-37 v-47+3 \mu(15-v)(1+v)^{2}}{49+41 v-2 v^{2}}
$$

solves $\frac{\partial e_{5}}{\partial a}=0$ and

$$
\begin{aligned}
c_{5 a}= & 15-v^{2}+15 v>0 \\
c_{5 b}= & 2(1-v)\left(14 v^{2}+28 v+19-v^{3}\right) \\
& +(1-\mu)\left(13 v^{3}+40 v^{2}+49 v+11-v^{4}\right) \geq 0 .
\end{aligned}
$$

Hence $e_{5} \geq 0$. As a result, $s_{5} \geq 0$ and thus $\mathbf{I}_{3} \leq 0$.

\section{Case $4: \mathrm{I}_{4} \leq 0$}

Here

$$
\begin{aligned}
n\left(\mathbf{A}_{4}\right)= & -4(M \mu p-M+p-1)^{2} s_{6}, \\
s_{6}= & 2 p-2+\left(3 \mu+6 p-3-4 \mu p-2 p^{2}\right) M \\
& +\left(4 \mu p^{2}-5 \mu p+3 \mu-2 p\right) M^{2}+(1-\mu p) M^{3}
\end{aligned}
$$

Then, substituting $M=2+\delta$,

$$
\begin{aligned}
\frac{\partial s_{6}}{\partial \delta}= & {[5(1-\mu)+2(1-p)(p+2+10 \mu-8 \mu p)] } \\
& +[8(1-\mu)+2(1-p)(2+7 \mu-4 \mu p)] \delta \geq 0
\end{aligned}
$$

and as a result for $\delta \geq 0$ we have

$$
s_{6} \geq\left. s_{6}\right|_{\delta=0}=2(3-2 p)[p(1-\mu)+\mu(1-3 p)] \geq 0 .
$$

\section{Case $5: I_{5} \leq 0$}

Here

$$
n\left(\mathbf{A}_{5}\right)=-s_{7} .
$$

We need to show that $s_{7} \geq 0$ when $X_{1} \leq 0$ and $X_{3} \geq 0$.

Since $X_{1} \leq 0$, we have $2 M p \mu \leq M+1$. Together with $X_{3} \geq 0$ this implies

$$
0 \leq n\left(X_{3}\right)=2 M p \mu-(M+1)-a(M+1)+2 p \leq-a(M+1)+2 p
$$


whence

$$
a \leq \frac{2 p}{M+1}
$$

Let us show that for this $a$ we have $s_{7} \geq 0$; substitute $a=b \cdot \frac{2 p}{M+1}$, where $b \in[0,1]$.

First, let $M=2$, then $\mu=\frac{1+a}{2}, p \in[3 / 4,1)$, and $s_{7}=\frac{3-2 p}{27} s_{8}$ where

$$
\begin{aligned}
s_{8}= & 512 b^{3} p^{8}-2688 b^{3} p^{7}+5760 b^{3} p^{6}+3456 b^{2} p^{7} \\
& -6912 b^{3} p^{5}-12672 b^{2} p^{6}+5184 b^{3} p^{4} \\
& +16416 b^{2} p^{5}+5184 b p^{6}-1944 b^{3} p^{3}-11664 b^{2} p^{4} \\
& -10368 b p^{5}+7776 b^{2} p^{3}+1728 p^{5}-2916 b^{2} p^{2} \\
& +11664 b p^{3}-11664 b p^{2}-7776 p^{3}+4374 b p+17496 p^{2}-17496 p+6561
\end{aligned}
$$

Note that we can write $s_{8}=e_{1}+e_{2}(1-p)+e_{3}(1-p)^{2}$, where

$$
\begin{aligned}
128 e_{1}= & \left(9-v^{2}-6 v\right)\left(81-v^{3}-9 v^{2}-63 v\right)\left(v^{3}+15 v^{2}+81-9 v\right)>0 \\
128 e_{2}= & 3\left(9-v^{2}\right)\left(v^{6}+21 v^{5}+168 v^{4}+666 v^{3}+81 v^{2}+81 v+486\right)>0 \\
64\left(e_{1}+e_{3}\right)= & {\left[2 v^{8}+33 v^{7}+234 v^{6}+783 v^{5}\right] } \\
& +\left[-648 v^{4}-6561 v^{3}+30618 v^{2}-28431 v+13122\right] \\
\geq & -648 v^{4}-6561 v^{3}+30618 v^{2}-28431 v+13122 \\
\geq & -1000 v^{4}-7000 v^{3}+24000 v^{2}-29000 v+13000 \\
= & 1000(1-v)\left(5+8(1-v)^{2}+v^{3}\right) \geq 0 .
\end{aligned}
$$

with $p=\frac{3+v}{4}, v \in[0,1]$. Consequently, since $(1-p)^{2}<1$ and $e_{1}>0$,

$$
s_{8}=e_{1}+e_{2}(1-p)+e_{3}(1-p)^{2} \geq e_{2}(1-p)+\left(e_{1}+e_{3}\right)(1-p)^{2} \geq 0
$$

and thus $s_{7} \geq 0$ as required.

For $M \geq 3$, set $M=3+\delta, \delta \geq 0$. Then

$$
s_{7}=\sum_{i=0}^{9} e_{i+1} \delta^{i}
$$

where

$$
\begin{aligned}
e_{1}= & 196608+(98304 b-393216) p \\
& +\left(-49152 b^{2}-442368 \mu^{2}-196608 b-737280 \mu+589824\right) p^{2} \\
& +\left(-24576 b^{3}+221184 b \mu^{2}+98304 b^{2}\right. \\
& \left.+1990656 \mu^{2}+294912 b+663552 \mu-540672\right) p^{3} \\
& +\left(49152 b^{3}+73728 b^{2} \mu-552960 b \mu^{2}-331776 \mu^{3}\right. \\
& -147456 b^{2}-2322432 \mu^{2}-270336 b \\
& +110592 \mu+233472) p^{4} \\
& +\left(-83968 b^{3}+184320 b^{2} \mu-55296 b \mu^{2}+774144 \mu^{3}\right.
\end{aligned}
$$




$$
\begin{aligned}
& +135168 b^{2}+1050624 \mu^{2}+116736 b \\
& -239616 \mu-36864) p^{5} \\
& +\left(52224 b^{3}-175104 b^{2} \mu+165888 b \mu^{2}-331776 \mu^{3}\right. \\
& \left.-58368 b^{2}-165888 \mu^{2}-18432 b+55296 \mu\right) p^{6} \\
& +\left(-9216 b^{3}+27648 b^{2} \mu+9216 b^{2}\right) p^{7} \\
& e_{2}=393216+(172032 b-540672) p \\
& +\left(-73728 b^{2}-958464 \mu^{2}-221184 b-2088960 \mu+835584\right) p^{2} \\
& +\left(-30720 b^{3}+423936 b \mu^{2}+86016 b^{2}+4589568 \mu^{2}\right. \\
& +344064 b+1898496 \mu-823296) p^{3} \\
& +\left(30720 b^{3}+282624 b^{2} \mu-1308672 b \mu^{2}-663552 \mu^{3}\right. \\
& -135168 b^{2}-5031936 \mu^{2}-344064 b \\
& -18432 \mu+344064) p^{4} \\
& +\left(-77312 b^{3}+181248 b^{2} \mu+4608 b \mu^{2}+1658880 \mu^{3}\right. \\
& +138240 b^{2}+2068992 \mu^{2}+142848 b \\
& -360960 \mu-49152) p^{5} \\
& +\left(50176 b^{3}-228864 b^{2} \mu+290304 b \mu^{2}-691200 \mu^{3}\right. \\
& \left.-56832 b^{2}-290304 \mu^{2}-19968 b+78336 \mu\right) p^{6} \\
& +\left(-7680 b^{3}+32256 b^{2} \mu+7680 b^{2}\right) p^{7} \\
& e_{3}=344064+(129024 b-208896) p+\left(-46080 b^{2}-906240 \mu^{2}\right. \\
& -49152 b-2558976 \mu+430080) p^{2} \\
& +\left(-15360 b^{3}+347136 b \mu^{2}+3072 b^{2}+4718592 \mu^{2}\right. \\
& +129024 b+2217984 \mu-522240) p^{3} \\
& +\left(-6144 b^{3}+334848 b^{2} \mu-1337856 b \mu^{2}-566784 \mu^{3}\right. \\
& -30720 b^{2}-4778496 \mu^{2}-175104 b \\
& -198144 \mu+210432) p^{4} \\
& +\left(-24448 b^{3}+42240 b^{2} \mu+100992 b \mu^{2}+1543680 \mu^{3}\right. \\
& +52992 b^{2}+1744512 \mu^{2}+69504 b \\
& -223872 \mu-26112) p^{5} \\
& +\left(17856 b^{3}-118464 b^{2} \mu+210816 b \mu^{2}-615168 \mu^{3}\right. \\
& \left.-20544 b^{2}-210816 \mu^{2}-8064 b+44160 \mu\right) p^{6} \\
& +\left(-2112 b^{3}+14016 b^{2} \mu+2112 b^{2}\right) p^{7} \\
& e_{4}=172032+(53760 b+64512) p \\
& +\left(-15360 b^{2}-488448 \mu^{2}+44544 b-1790976 \mu+64512\right) p^{2} \\
& +\left(-3840 b^{3}+157440 b \mu^{2}-23040 b^{2}\right. \\
& \left.+2843904 \mu^{2}+1416960 \mu-176640\right) p^{3}
\end{aligned}
$$




$$
\begin{aligned}
& +\left(-9984 b^{3}+193536 b^{2} \mu-772608 b \mu^{2}\right. \\
& -268032 \mu^{3}+7680 b^{2}-2598912 \mu^{2}-44544 b \\
& -170496 \mu+68352) p^{4} \\
& +\left(93024 b \mu^{2}-13632 b^{2} \mu+32 \mu\left(25448 \mu^{2}+25515 \mu-2283\right)\right. \\
& \left.-32 b\left(77 b^{2}-282 b-525\right)-6912\right) p^{5} \\
& +\left(2784 b^{3}-30336 b^{2} \mu+81312 b \mu^{2}-303168 \mu^{3}\right. \\
& \left.-3264 b^{2}-81312 \mu^{2}-1440 b+12384 \mu\right) p^{6} \\
& +\left(-192 b^{3}+2688 b^{2} \mu+192 b^{2}\right) p^{7} \\
& e_{5}=53760+(13440 b+91392) p+\left(-2880 b^{2}\right. \\
& \left.-164160 \mu^{2}+34560 b-792768 \mu-26880\right) p^{2} \\
& +\left(-480 b^{3}+42720 b \mu^{2}-11520 b^{2}\right. \\
& \left.+1109088 \mu^{2}-13440 b+548640 \mu-33600\right) p^{3} \\
& +\left(62496 b^{2} \mu-275952 b \mu^{2}-885744 \mu^{2}-67536 \mu\right. \\
& \left.-75792 \mu^{3}-96 b\left(34 b^{2}-50 b+59\right)+12432\right) p^{4} \\
& +\left(160 b^{3}-8544 b^{2} \mu+38928 b \mu^{2}+266192 \mu^{3}+576 b^{2}\right. \\
& \left.+229104 \mu^{2}+2016 b-13200 \mu-912\right) p^{5} \\
& +\left(160 b^{3}-3840 b^{2} \mu+17568 b \mu^{2}-89344 \mu^{3}-192 b^{2}\right. \\
& \left.-17568 \mu^{2}-96 b+1728 \mu\right) p^{6}+192 b^{2} \mu p^{7} \\
& e_{6}=10752+(2016 b+38976) p \\
& +\left(-288 b^{2}-35232 \mu^{2}+10848 b-230880 \mu-14784\right) p^{2} \\
& +\left(-24 b^{3}+6936 b \mu^{2}-2544 b^{2}+290664 \mu^{2}\right. \\
& -4032 b+132888 \mu-3408) p^{3} \\
& +\left(11568 b^{2} \mu-456 b^{3}-62472 b \mu^{2}-12816 \mu^{3}+816 b^{2}\right. \\
& \left.-193752 \mu^{2}-288 b-14328 \mu+1200\right) p^{4} \\
& +\left(32 b^{3}-1536 b^{2} \mu+8688 b \mu^{2}+55168 \mu^{3}\right. \\
& \left.+38544 \mu^{2}+96 b-1248 \mu-48\right) p^{5} \\
& +\left(-192 b^{2} \mu+2016 b \mu^{2}-15744 \mu^{3}-2016 \mu^{2}+96 \mu\right) p^{6} \\
& e_{7}=1344+(168 b+9072) p+\left(-12 b^{2}-4716 \mu^{2}\right. \\
& +1824 b-44340 \mu-3024) p^{2} \\
& +\left(624 b \mu^{2}-276 b^{2}+51252 \mu^{2}-504 b+19764 \mu-144\right) p^{3} \\
& +\left(-24 b^{3}+1152 b^{2} \mu-8760 b \mu^{2}-1200 \mu^{3}\right. \\
& \left.+48 b^{2}-26568 \mu^{2}-1584 \mu+48\right) p^{4} \\
& +\left(-96 b^{2} \mu+1008 b \mu^{2}+7072 \mu^{3}+3600 \mu^{2}-48 \mu\right) p^{5} \\
& +\left(96 b \mu^{2}-1536 \mu^{3}-96 \mu^{2}\right) p^{6} \\
& e_{8}=96+(6 b+1236) p+\left(-360 \mu^{2}+162 b-5424 \mu-300\right) p^{2}
\end{aligned}
$$




$$
\begin{aligned}
& +\left(24 b \mu^{2}-12 b^{2}+5868 \mu^{2}-24 b+1656 \mu\right) p^{3} \\
& +\left(48 b^{2} \mu-696 b \mu^{2}-48 \mu^{3}-2088 \mu^{2}-72 \mu\right) p^{4} \\
& +\left(48 b \mu^{2}+512 \mu^{3}+144 \mu^{2}\right) p^{5}-64 \mu^{3} p^{6}
\end{aligned}
$$

and the expressions for $e_{9}$ and $e_{10}$ are given a little bit further.

First, we will show that $e_{i} \geq 0, i=1, \ldots, 8$.

\section{Proof that $e_{1}, \ldots, e_{8}>0$}

It turns out that it is easiest is to use a computer-assisted proof in this case; to this end we developed the method which we call a Box method; it may have been described by other authors, but since we do not have the reference to the right source, we give its description below.

First of all, we substitute

$$
p=\frac{1+x_{1}}{2}, b=x_{2}, \mu=x_{3} ; \quad x_{i} \in[0,1], i=1,2,3 .
$$

Let $\mathrm{m}=\min _{a_{i} \leq x_{i} \leq b_{i}, i=1,2,3} f\left(x_{1}, x_{2}, x_{3}\right)$ where

$$
f\left(x_{1}, x_{2}, x_{3}\right)=f_{+}\left(x_{1}, x_{2}, x_{3}\right)-f_{-}\left(x_{1}, x_{2}, x_{3}\right)
$$

and $f_{+}$and $f_{-}$are polynomials with non-negative coefficients. We want to show that $\mathrm{m}>0$.

Let

$$
G_{f ; M}=\min _{i_{1}, i_{2}, i_{3}=0, \ldots, M-1}\left[f_{+}\left(\frac{i_{1}}{M}, \frac{i_{2}}{M}, \frac{i_{3}}{M}\right)-f_{-}\left(\frac{i_{1}+1}{M}, \frac{i_{2}+1}{M}, \frac{i_{3}+1}{M}\right)\right] .
$$

Since

$$
\mathrm{m} \geq G_{f ; M} \rightarrow \mathrm{m}
$$

as $M \rightarrow \infty$, we conclude that $\mathrm{m}>0$ if and only if $G_{f, M} \geq 0$ for some $M \geq 1$. Checking that $G_{f, M} \geq 0$ can be quite tedious and time-consuming for large $M$, however, this could be easily accomplished with the help of a computer; please note, that the results are still completely rigorous, unlike e.g. simulations.

The results of application of this method to $e_{1}, \ldots, e_{8}$ are presented in the following table:

$$
\begin{aligned}
& G_{e_{1}, 2000}>825, \quad G_{e_{2}, 500}>25, \quad G_{e_{3}, 400}>1860, \quad G_{e_{4}, 300}>2397, \\
& G_{e_{5}, 200}>672, \quad G_{e_{6}, 200}>148, \quad G_{e_{7}, 200}>5, \quad G_{e_{8}, 400}>3 .
\end{aligned}
$$

Consequently, $e_{j}>0$ for all $j=1, \ldots, 8$.

\section{Proof that $e_{9} \geq 0$ and $e_{10} \geq 0$}

The Box method of the previous section would not work for $e_{9}$ and $e_{10}$, since these functions do touch zero in the required area, and hence the minimum is, in fact, 0 . Therefore, we have to handle these two cases analytically.

We have

$$
\begin{aligned}
e_{9}= & 4 p^{2} \mu\left(4 \mu^{2} p^{3}-18 \mu p^{2}+99 \mu p-3+15 p-96\right) \\
& -12 p^{2}+93 p+3+\left[6 p^{2}(1-2 \mu p)(2 \mu p+1)\right] b,
\end{aligned}
$$


hence, the minimum is achieved either at $b=0$ or $b=1$.

For $\mu<1 /(2 p)$ we have $e_{9} \geq e_{9 a}$, where

$$
\begin{aligned}
e_{9 a} & =\left.e_{9}\right|_{b=0}=2 s^{3} p^{2}-18 p^{2} s^{2}+30 s p^{2}+99 s^{2} p-12 p^{2}-192 p s-3 s^{2}+93 p+3 \\
& =2 p^{2}+(1-s)\left[6(1-p)+(1-s)\left(99 p+2 p^{2} s-14 p^{2}-3\right)\right] \geq 0
\end{aligned}
$$

where $s=2 p \mu \in[0,1]$.

In case $\mu \geq 1 /(2 p)$ we have $e_{9} \geq e_{9 b}$, where

$$
\begin{aligned}
e_{9 b}= & \left.e_{9}\right|_{b=1}=16 p^{5} s^{3}-24 p^{4} s^{3}-72 p^{4} s^{2}+12 p^{3} s^{3} \\
& +468 p^{3} s^{2}-2 s^{3} p^{2}-24 p^{3} s-426 p^{2} s^{2} \\
& +24 s p^{2}+111 s^{2} p+2 p^{2}-18 p s-3 s^{2}+6 s
\end{aligned}
$$

where $\mu=\frac{1}{2 p}+s\left(1-\frac{1}{2 p}\right), s \in[0,1]$. Now,

$$
\frac{\partial^{2}}{\partial s^{2}} e_{9 b}=6(2 p-1)^{2}\left(14+(2 p-1)\left(2 p^{2} s-3 p+15\right)\right) \geq 0
$$

so the minimum of $e_{9 b}$ w.r.t. $s$ is achieved where $\frac{\partial}{\partial s} e_{9 b}=0$, i.e.

$$
s_{c r}=\frac{6 p^{2}-33 p+1+R}{2 p^{2}(2 p-1)}, \text { where } R=\sqrt{44 p^{4}-400 p^{3}+1105 p^{2}-66 p+1}
$$

and equals

$$
\begin{aligned}
& \frac{3996 p^{5}-284 p^{6}-19956 p^{4}+37329 p^{3}-3291 p^{2}+99 p-1+\left(400 p^{3}-44 p^{4}-1105 p^{2}+66 p-1\right) R}{2 p^{4}} \\
& \geq 22120.5-1576 \sqrt{197}=0.285896>0
\end{aligned}
$$

for $p \geq 1 / 2$.

Finally, trivially, we have $e_{10}=3 p(2 \mu p-1)^{2} \geq 0$. Consequently, $s_{7} \geq 0$ and $\mathbf{I}_{5} \leq 0$.

Combining this with the previously established inequalities $\mathbf{I}_{j} \leq 0, j=1,2,3,4$, we complete the proof Lemma 2.

\section{References}

1. De Giorgi, E., Reimann, S.: The $\alpha$-beauty contest: choosing numbers, thinking intervals. Games Econ. Behav. 64, 470-486 (2008)

2. Grinfeld, M., Knight, P.A., Wade, A.R.: Rank-driven Markov processes. J. Stat. Phys. 146, 378-407 (2012)

3. Grinfeld, M., Volkov, S., Wade, A.R.: Convergence in a multidimensional randomized Keynesian beauty contest. Adv. Appl. Probab. 47, 57-82 (2015)

4. Kennerberg, P., Volkov, S.: Jante's law process. Adv. Appl. Probab. 50, 414-439 (2018)

5. Keynes, J.M.: The General Theory of Employment. Interest and Money. Macmillan, London (1936)

6. Moulin, H.: Game Theory for the Social Sciences, 2nd edn. New York University Press, New York (1986)

7. Seel, C., Tsakas, E.: Rationalizability and Nash equilibria in guessing games. Games Econ. Behav. 106, 75-88 (2017)

8. Williams, D.: Probability with Martingales. Cambridge University Press, Cambridge (1991)

Publisher's Note Springer Nature remains neutral with regard to jurisdictional claims in published maps and institutional affiliations. 